University of Nebraska - Lincoln

DigitalCommons@University of Nebraska - Lincoln

$11-15-2013$

\title{
Resonant enhancement of the harmonic-generation spectrum of beryllium
}

Jean Marcel Ngoko Djiokap

University of Nebraska-Lincoln, marcelngoko@unl.edu

Anthony F. Starace

University of Nebraska-Lincoln, astarace1@unl.edu

Follow this and additional works at: https://digitalcommons.unl.edu/physicsstarace

Part of the Atomic, Molecular and Optical Physics Commons, Elementary Particles and Fields and String Theory Commons, and the Plasma and Beam Physics Commons

Ngoko Djiokap, Jean Marcel and Starace, Anthony F., "Resonant enhancement of the harmonic-generation spectrum of beryllium" (2013). Anthony F. Starace Publications. 202.

https://digitalcommons.unl.edu/physicsstarace/202

This Article is brought to you for free and open access by the Research Papers in Physics and Astronomy at DigitalCommons@University of Nebraska - Lincoln. It has been accepted for inclusion in Anthony F. Starace Publications by an authorized administrator of DigitalCommons@University of Nebraska - Lincoln. 


\title{
Resonant enhancement of the harmonic-generation spectrum of beryllium
}

\author{
J. M. Ngoko Djiokap and Anthony F. Starace \\ Department of Physics and Astronomy, The University of Nebraska, Lincoln, Nebraska 68588-0299, USA
}

(Received 18 September 2013; published 15 November 2013)

\begin{abstract}
The high-order harmonic-generation ( $\mathrm{HHG}$ ) spectrum of $\mathrm{Be}$ is investigated in the multiphoton regime by solving the full-dimensional, two-active-electron, time-dependent Schrödinger equation in an intense $\left(\approx 10^{13} \mathrm{~W} / \mathrm{cm}^{2}\right)$, 30-cycle laser field. As the laser frequency $\omega_{L}$ varies from 1.7 to $1.8 \mathrm{eV}$ (which is in the tunable range of a Ti:sapphire laser), the seventh harmonic becomes resonant sequentially with the transition between the ground state and two doubly excited autoionizing states, $2 p 4 s\left({ }^{1} P\right)\left(\right.$ at $\left.\omega_{L}=1.734 \mathrm{eV}\right)$ and $2 p 5 s\left({ }^{1} P\right)$ (at $\omega_{L}=1.785 \mathrm{eV}$ ), while the third harmonic becomes resonant with the $2 s 2 p\left({ }^{1} P\right)$ singly excited state (at $\omega_{L}=1.766 \mathrm{eV}$ ). At each of these resonant frequencies, the HHG power spectrum is found to increase by an order of magnitude over a range of harmonics that form a plateau, extending from the resonant harmonic up to a cutoff at the 25th harmonic. In contrast to the well-known rescattering plateau cutoff law appropriate in the tunneling regime (which predicts a cutoff at the fifth or seventh harmonic), the multiphoton regime plateau we find for Be originates from atomic resonance effects. Off resonance, the Be HHG spectrum decreases monotonically with harmonic order. By taking the ratio of the integrated harmonic power of the seventh harmonic to that of the fifth harmonic, one can isolate the resonant effects of the two doubly excited states in the HHG spectrum from those of singly excited resonance states. These ratios exhibit resonance profiles for driving laser-pulse durations much longer than the lifetimes of these autoionizing states. The energy widths of these resonance features are comparable to the widths of the laser pulse and are much smaller than the autoionizing state widths. These results demonstrate an important role for electron correlations in enhancing harmonic-generation rates in the multiphoton regime.
\end{abstract}

DOI: 10.1103/PhysRevA.88.053412

PACS number(s): 32.80.Rm, 42.65.Ky, 32.80.Zb

\section{INTRODUCTION}

The effects of multielectron correlations on nonlinear processes are of great interest in strong-field physics for obtaining a deeper understanding of laser-matter interactions [1]. Since its discovery in the late 1980s [2,3], high-order harmonic generation (HHG) has been among the most intensively investigated laser-driven nonlinear processes in strong-field physics. In large part, this high interest is due to its many important applications, such as in producing attosecond light pulses [4-12], in producing coherent soft $x$ rays with energies in the water window region and beyond [13-16], and in determining atomic and molecular properties and structures [17-23]. For two decades the "three-step" scenario [24-26] has served a crucial role in understanding and planning HHG experiments. In this scenario, in the first step a single active electron in an atom or molecule is ionized by an intense laser field by tunneling; in the second step, the active electron is driven away from and then (when the laser field changes sign) back to its parent ion; in the third step, the electron may then recombine (when the laser field is changing sign) with its parent ion by emitting a harmonic photon. This simple model has led to the ad hoc assumption that the HHG spectrum of an atom or molecule should be proportional to the field-free photoionization cross section of the atom or molecule (which is simply related to the photorecombination cross section by the principle of detailed balance [27]). This close connection between an atom or molecule's HHG spectrum and its field-free photoionization cross section has been well-demonstrated numerically by the so-called quantitative rescattering (QRS) theory of Lin and collaborators [18-20]. Recently, an analytical derivation of this close connection was obtained for a simple model system $[28,29]$. Specifically, a closed-form analytic expression of the $\mathrm{HHG}$ rate for a single-electron model system was shown to be factorizable in terms of three factors, with each corresponding to one of the steps of the three-step scenario. The factors corresponding to the tunnel ionization and the photorecombination steps, of course, are dependent on the particular parameters of the target system, while the factor corresponding to the second step was shown to depend only on laser field parameters.

These numerical [18-20] and analytical [28,29] demonstrations of the proportionality of HHG rates to field-free photoionization cross sections provide a means to include many-electron correlation effects on HHG rates: one can employ accurate photoionization cross-section data (either calculated numerically with inclusion of many-electron correlations or taken from experiment) when calculating HHG rates. This is the basis of the QRS theory for molecular HHG [18-20]. A dramatic example for atoms was the prediction that the HHG rates for the $\mathrm{Xe}$ atom from its valence $5 p$ subshell would be strongly influenced (through interchannel many-electron correlations) by the well-known giant dipole resonance in the $4 d$-subshell photoionization cross section of $\mathrm{Xe}$ [30]. This prediction was subsequently confirmed experimentally [31]. Similarly, strong resonances in experimental HHG spectra of the $\mathrm{Cr}^{+}$and $\mathrm{Mn}^{+}$transition-metal ions [32,33] were shown theoretically to originate from well-known intense $3 p \rightarrow 3 d$ inner-shell transitions in the photoionization cross sections of those ions [34].

The $a b$ initio calculation of HHG rates with inclusion of many-electron correlations is at present not possible in the tunneling regime for which the above-described three-step scenario is applicable. The tunneling regime is usually defined by a Keldysh parameter [35] $\gamma<1$, where $\gamma \equiv \sqrt{I_{0} / 2 U_{p}}, I_{0}$ 
is the binding energy of the atom, and $U_{p}$ is the ponderomotive potential energy of the active electron in the driving laser field. In atomic units, $U_{p} \equiv\left(E_{0} / 2 \omega_{L}\right)^{2}$, where $E_{0}$ is the amplitude and $\omega_{L}$ is the frequency of the laser field. Thus the tunneling regime corresponds to a large ponderomotive potential, i.e., to intense laser field strengths and/or small laser frequencies. These parameters cause difficulty for $a b$ initio calculations involving many-electron correlations: intense laser fields lead to large electron excursions in coordinate space, while small laser frequencies imply absorption and/or emission of many photons, requiring the inclusion of a large number of orbital angular momenta. For this reason, ab initio calculations for many-electron atoms interacting with laser fields have in general been carried out in the multiphoton regime, defined by a Keldysh parameter $\gamma>1$, i.e., for large laser frequencies and small field amplitudes.

Relatively few $a b$ initio calculations of HHG spectra of atoms with inclusion of many-electron correlations have been reported. These investigations have focused primarily on the HHG spectrum of the $\mathrm{He}$ atom [36-39], although recently HHG results have been reported also for the $\mathrm{Ar}^{+}$ion $[40,41]$. Various theoretical formulations have been employed: Guan et al. [36] use a time-dependent generalized pseudospectral approach in hyperspherical coordinates; Gilary et al. [37] use a Floquet-type formulation; we have solved the time-dependent, two-electron Schrödinger equation by means of an eigenstate expansion within a finite radial box [38] (see also Refs. [42, 43]); and Brown et al. [39-41] employ a time-dependent $R$ matrix approach. In all cases the HHG spectra are calculated in the multiphoton regime for laser wavelengths (respectively, photon energies) from $185 \mathrm{~nm}(6.7 \mathrm{eV})$ to $390 \mathrm{~nm}(3.2 \mathrm{eV})$ and intensities of the order of $10^{14} \mathrm{~W} / \mathrm{cm}^{2}$.

Since calculations of HHG spectra are typically carried out for a fixed driving laser frequency (because that is the way experimental measurements are carried out), the discovery of resonance effects in HHG spectra is often accidental, especially since the exact energy location of target bound or autoionizing states in an intense laser field is not known. Nevertheless, in carrying out an $a b$ initio calculation for a $\mathrm{KrF}$ laser wavelength of $248.6 \mathrm{~nm}$ and a driving laser intensity of $10^{14} \mathrm{~W} / \mathrm{cm}^{2}$, Guan et al. [36] found effects of a group of doubly excited states located below $65 \mathrm{eV}$ in the HHG spectrum of He. In our prior work [38], we focused specifically on determining the effect of the well-isolated $2 s 2 p\left({ }^{1} P\right)$ autoionizing state (located $\approx 60 \mathrm{eV}$ above the ground state) on the He HHG spectrum. In order to ensure that resonant transitions to this state in the driving laser field would occur, we carried out our calculations for a range of photon energies from $4.4 \mathrm{eV}$ to $6.6 \mathrm{eV}$ and found resonant enhancement of the HHG rates for the 13th, 11th, and ninth harmonics [38]. Recently, Brown et al. [40] investigated the influence of the Ar autoionizing states $3 s 3 p^{6} n \ell$ on the fifth harmonic for driving laser fields with wavelengths in the range of 200-240 nm. They demonstrated asymmetric harmonic line shapes (as a function of laser wavelength) in the vicinity of these autoionizing states. As far as we are aware, however, the influence of resonant doubly excited states on the entire HHG spectrum of a particular target atom has not been investigated. Moreover, none of the above-cited ab initio calculations have been carried out for the Ti:sapphire laser field that is the most commonly used one in experiments. Finally, the only experiment of which we are aware that shows evidence of a doubly excited state on an HHG spectrum is that of Gilbertson et al. [44] by means of the double optical gating (DOG) technique. They found a prominent feature near $\approx 60 \mathrm{eV}$ in the harmonic supercontinuum spectrum of He that was attributed to the $2 s 2 p\left({ }^{1} P\right)$ autoionizing state. However, the driving laser parameters in that experiment [44] were in the tunneling regime (specifically, they employed a photon energy $\omega=1.55 \mathrm{eV}$ and an intensity $I=10^{15} \mathrm{~W} / \mathrm{cm}^{2}$ ), which for the reasons given above are at present a difficult regime for $a b$ initio theoretical calculations that incorporate many-electron correlations.

In this paper we present numerical results for the HHG spectrum of beryllium (Be), which we treat as a two-activeelectron system. Compared to $\mathrm{He}$, the $\mathrm{Be}$ atom is advantageous because its singly and doubly excited field-free energy spectra lie much closer to its ground state. Hence only a few photons (with frequencies near those of the tunable Ti:sapphire laser) are required to reach its doubly excited levels from the ground state. Moreover, its excited states do not have the degeneracies typical of the He atom. Therefore, Be appears to be an excellent testbed for numerical calculations that account for two-electron correlations rigorously for laser frequencies commonly available experimentally. We note that for the lowest-order process involving the interaction of the $\mathrm{Be}$ atom with light, i.e., atomic photoionization, Be has for many decades been investigated using a variety of accurate theoretical approaches [45-54]. There have also been a number of accurate experimental measurements showing the strong influence of autoionizing state resonances on its photoionization cross section [55-59]. Recently, a theoretical pump-probe investigation of the decay of these autoionizing resonances has been carried out [60]. Also, a correlated proof-of-principle calculation of HHG for a one-dimensional (1D) model $\mathrm{Be}$ atom has been presented [61]. However, for high-order, nonperturbative interactions with light, there have not been (as far as we are aware) any 3D theoretical calculations including effects of electron correlations. The only experimental HHG measurements, moreover, concerned Be plasma plumes, which comprised singly and doubly ionized Be ions and not the neutral atom [62].

The goal of this paper is to investigate the influence of two broad autoionizing resonances, $2 p 4 s\left({ }^{1} \mathrm{P}\right)$ and $2 p 5 s\left({ }^{1} \mathrm{P}\right)$, on the HHG spectrum of Be by solving in its full dimensionality the two-active-electron, time-dependent Schrödinger equation (TDSE) with inclusion of electron correlations. We note that the lifetimes of these two resonances (respectively, $3.78 \mathrm{fs}$ and $8.55 \mathrm{fs}$ ) are about five and two times shorter than the $17.7 \mathrm{fs}$ lifetime of the He $2 s 2 p\left({ }^{1} P\right)$ doubly excited state investigated in Ref. [38]. In Sec. II we describe briefly our theoretical formulation and numerical approach for describing the fieldfree atomic structure of the Be atom and for calculating the HHG spectrum. In Sec. III, we present our numerical results and analyses for the HHG spectrum of Be, with an emphasis on the role of resonant doubly excited autoionizing states. Finally, in Sec. IV, we briefly summarize our results and draw some conclusions. We employ atomic units (a.u.), i.e., $e=$ $\hbar=m=1, c=1 / \alpha$, throughout this paper unless otherwise stated. 


\section{THEORETICAL FORMULATION AND NUMERICAL APPROACH}

In this section, we present our theoretical formulation and numerical approach for obtaining the field-free atomic structure and the harmonic-generation spectrum of Be. Our numerical methods are similar to those employed to treat the effect of the $2 s 2 p\left({ }^{1} P\right)$ doubly excited state on the HHG spectrum of $\mathrm{He}$ [38] and laser detachment of the lithium negative ion $[42,43]$, modified appropriately, of course, to describe the $\mathrm{Be}$ atom.

\section{A. Calculation of Be field-free atomic structure}

We treat $\mathrm{Be}$ as a two-active-electron system with each of the valence-shell electrons assumed to move in a potential $V(r)$ describing the $\mathrm{Be}^{2+}$ core. We adopt the following form of the potential $V(r)$ :

$$
\begin{aligned}
V(r)= & -\frac{1}{r}\left[\left(Z-Z_{c}\right)+Z_{c}(1+\beta r) e^{-2 \beta r}\right] \\
& -\frac{\alpha_{c}}{2 r^{4}}\left(1-e^{-\left(r / r_{c}\right)^{3}}\right)^{2},
\end{aligned}
$$

where $Z=4$ denotes the nuclear charge, $Z_{c}=2$ denotes the number of electrons in the $\mathrm{Be}^{2+}$ core, $\alpha_{c}$ is the polarizability for the $\mathrm{Be}^{2+}$ core (where we use the value $\alpha_{c}=0.05182$ given in Ref. [63]), and the values of the exponential parameters $\beta=2.333710$ and $r_{c}=0.4$ are taken respectively from Refs. [64] and [54] in which they are determined by optimizing fits to experimental energy-level data for the $\mathrm{Be}^{+}$ion. We note that the potential $V(r)$ in Eq. (1) has the correct Coulombic forms at the radial boundaries: $V(r) \rightarrow-Z / r$ as $r \rightarrow 0$ and $V(r) \rightarrow-\left(Z-Z_{c}\right) / r$ as $r \rightarrow \infty$. The Schrödinger equation describing the radial wave function $R_{n l}(r)$ of the valence electron in the $\mathrm{Be}^{+}$ion (having orbital angular momentum $l$, radial quantum number $n$, and energy $\left.\epsilon_{n l}\right)$ is

$$
\left[-\frac{1}{2} \frac{d^{2}}{d r^{2}}+\frac{l(l+1)}{2 r^{2}}+V(r)\right] R_{n l}(r)=\epsilon_{n l} R_{n l}(r) .
$$

The radial wave functions $R_{n l}(r)$ are evaluated on a mesh having 2500 points (for both $\epsilon_{n l} \leqslant 0$ and $\epsilon_{n l}>0$ ) by solving Eq. (2) in a radial box of size $r \equiv r_{0}=75$ a.u., with the following boundary conditions $[38,42,43]: R_{n l}\left(r_{0}\right)=0$. To solve Eq. (2), we transform the latter second-order differential equation into a set of two coupled first-order differential equations, which are solved by combining a Runge-Kutta method of order 4 with the Adams-Moulton predictor-corrector method. The Runge-Kutta method is used for the solution at smaller radial distances, where the short-range character of $V(r)$ is dominant. At larger distances the predictor-corrector method is used. Results for the field-free energy spectrum of the $\mathrm{Be}^{+}$ion are given in Table I. One sees that there is good agreement with the theoretical results of Refs. [54,64] and the experimental results of Ref. [65].

The bound and continuum one-electron orbitals $R_{n l}$ obtained serve as a complete basis (except for truncation) for the two-electron wave function

$$
\Psi\left(\mathbf{r}_{1}, \mathbf{r}_{2}\right)=\sum_{L M} \sum_{l_{1}, l_{2}} \sum_{n_{1}, n_{2}} \widetilde{\Psi}_{n_{1}, n_{2}}^{l_{1}, l_{2}, L M} \mathcal{A} F_{n_{1}, n_{2}}^{l_{1}, l_{2}, L M}\left(\mathbf{r}_{1}, \mathbf{r}_{2}\right),
$$

TABLE I. Energies of the $1 s^{2} n l$ states of $\mathrm{Be}^{+}$: (a) $\epsilon_{n l}$ (a.u.), relative to the ionization threshold of the $\mathrm{Be}^{+}$ion, and (b) $\epsilon_{e g}(n l) \equiv$ $\epsilon_{n l}-E_{g}(\mathrm{eV})$, the excitation energy relative to the ground-state energy $E_{g}$ of the Be atom (see Table II).

\begin{tabular}{lrrrrrr}
\hline \hline & \multicolumn{3}{c}{ (a) $\epsilon_{n l}($ a.u. $)$} & & \multirow{2}{*}{ (b) $\epsilon_{e g}(n l)(\mathrm{eV})$} \\
\cline { 2 - 4 }$n l$ & This work & Ref. [64] & Ref. [65] & & This work & Ref. [54] \\
\hline $2 s$ & -0.670116 & -0.669248 & -0.669246 & & 9.2951 & 9.2959 \\
$3 s$ & -0.267869 & -0.267685 & -0.267233 & & 20.241 & 20.2305 \\
$4 s$ & -0.143449 & -0.143381 & -0.143153 & & 23.626 & \\
$5 s$ & -0.089221 & -0.089196 & -0.089065 & & 25.102 & \\
$2 p$ & -0.524613 & -0.523718 & -0.523768 & & 13.254 & 13.2543 \\
$3 p$ & -0.230074 & -0.229798 & -0.229582 & & 21.269 & 21.2688 \\
$4 p$ & -0.128372 & -0.128255 & -0.128134 & & 24.037 & \\
$5 p$ & -0.081738 & -0.081687 & -0.081610 & & 25.306 & \\
$3 d$ & -0.222407 & -0.222404 & -0.222478 & & 21.478 & 21.4569 \\
$4 d$ & -0.125106 & -0.125103 & -0.125124 & & 24.126 & \\
$5 d$ & -0.080061 & -0.080018 & -0.080067 & & 25.351 & \\
\hline \hline
\end{tabular}

where $\mathbf{r}_{1}$ and $\mathbf{r}_{2}$ are the coordinates of the two electrons and $\widetilde{\Psi}_{n_{1}, n_{2}}^{l_{1}, l_{2}, L M}$ is the expansion coefficient of the two-electron basis state,

$$
F_{n_{1}, n_{2}}^{l_{1}, l_{2}, L M}\left(\mathbf{r}_{1}, \mathbf{r}_{2}\right)=\frac{R_{n_{1}, l_{1}}\left(r_{1}\right)}{r_{1}} \Lambda_{l_{1}, l_{2}}^{L M}\left(\hat{r}_{1}, \hat{r}_{2}\right) \frac{R_{n_{2}, l_{2}}\left(r_{2}\right)}{r_{2}},
$$

for total angular momentum $L$ and projection $M$. In Eq. (3), $\mathcal{A}=\left(1+P_{12}\right) / \sqrt{2}$ is the normalized antisymmetrization operator, in which the operator $P_{12}$ exchanges the electron states $\left(n_{1}, l_{1}\right)$ and $\left(n_{2}, l_{2}\right)$. Owing to electric dipole selection rules and the fact that the Be ground state is a singlet spin state, $\mathcal{A}$ ensures the symmetry of the spatial wave function under particle interchange, as required by the Pauli principle. The angular part of Eq. (4) is a bipolar harmonic,

$$
\Lambda_{l_{1}, l_{2}}^{L M}\left(\hat{r}_{1}, \hat{r}_{2}\right)=\sum_{m_{1}, m_{2}}\left\langle l_{1} m_{1} l_{2} m_{2} \mid L M\right\rangle Y_{l_{1}, m_{1}}\left(\hat{r}_{1}\right) Y_{l_{2}, m_{2}}\left(\hat{r}_{2}\right),
$$

which couples the two individual electron orbital angular momenta $l_{1}$ and $l_{2}$ in the $L S$-coupling scheme, where $\left\langle l_{1} m_{1} l_{2} m_{2} \mid L M\right\rangle$ is a Clebsch-Gordan coefficient and $Y_{l, m}(\hat{r})$ is a spherical harmonic. In order to preserve parity, which is a good quantum number, the orbital angular momenta of the electrons in laser-excited states must satisfy $(-1)^{L}=(-1)^{l_{1}+l_{2}}$ for interaction of the ${ }^{1} S$ ground state of Be with linearly polarized photons.

We employ ten values of the total angular momentum $L$, where $0 \leqslant L \leqslant 9$, and note that $L_{\max }=5$ has been found to be sufficient in HHG calculations for similar values of the Keldysh parameter [36,38]. For each total angular momentum $L$, we use four pairs of one-electron angular momenta $\left(l, l^{\prime}\right)$, i.e., for a given $L$ and a given value of $l$, where $0 \leqslant l \leqslant 6$, we chose $l^{\prime}$ according to the usual angular momentum and parity selection rules and, to avoid redundancy, $l \leqslant l^{\prime}$. The discrete set of eigenenergies for $\mathrm{Be}$ (corresponding to both bound states and to pseudostates representing the continuum) are obtained by representing the two-electron wave function as in Eq. (3) to solve the time-independent two-electron Schrödinger equation $H \Psi\left(\mathbf{r}_{1}, \mathbf{r}_{2}\right)=E \Psi\left(\mathbf{r}_{1}, \mathbf{r}_{2}\right)$, where $H\left(\mathbf{r}_{1}, \mathbf{r}_{2}\right)=h\left(\mathbf{r}_{1}\right)+h\left(\mathbf{r}_{2}\right)+$ $1 / r_{12}$ is the nonrelativistic Hamiltonian for $\mathrm{Be}$, and $h(\mathbf{r})$ is 
TABLE II. Energies of the $1 s^{2} 2 s n l$ states of Be: (a) $E_{e}(n l)$ (a.u.), relative to the Be double-ionization threshold, and (b) $E_{e g}(n l) \equiv$ $E_{e}(n l)-E_{g}(\mathrm{eV})$, the single-electron excitation energy relative to the Be $2 s^{2}\left({ }^{1} S\right)$ ground-state energy, $E_{g}$.

\begin{tabular}{|c|c|c|c|c|}
\hline \multirow[b]{2}{*}{$2 s n l$} & \multicolumn{3}{|c|}{ (a) $E_{e}(n l)$ (a.u.) } & \multirow{2}{*}{$\frac{\text { (b) } E_{e g}(\mathrm{eV})}{\text { This work }}$} \\
\hline & This work & Ref. [64] & Ref. [65] & \\
\hline $2 s^{2}\left({ }^{1} S\right)$ & -1.011704 & -1.007430 & -1.011850 & 0.000 \\
\hline $2 s 3 s\left({ }^{1} S\right)$ & -0.763455 & -0.761190 & -0.762720 & 6.7552 \\
\hline $2 s 4 s\left({ }^{1} S\right)$ & -0.715400 & -0.713920 & -0.714570 & 8.0628 \\
\hline $2 s 5 s\left({ }^{1} S\right)$ & -0.696848 & & & 8.5677 \\
\hline $2 s 2 p\left({ }^{1} P\right)$ & -0.816742 & -0.810060 & -0.817900 & 5.3052 \\
\hline $2 s 3 p\left({ }^{1} P\right)$ & -0.738004 & -0.734920 & -0.737610 & 7.4478 \\
\hline $2 s 4 p\left({ }^{1} P\right)$ & -0.707162 & -0.704370 & & 8.2871 \\
\hline $2 s 5 p\left({ }^{1} P\right)$ & -0.693181 & & & 8.6673 \\
\hline $2 s 6 p\left({ }^{1} P\right)$ & -0.685547 & & & 8.8749 \\
\hline $2 s 7 p\left({ }^{1} P\right)$ & -0.678763 & & & 9.0596 \\
\hline
\end{tabular}

the one-electron Hamiltonian whose radial part is given in Eq. (2). The multipole expansion of the electrostatic potential $1 / r_{12}$ [38] is used to treat electron correlations exactly.

The field-free energy spectrum of Be is given in Table II. With our choices of calculational parameters, we obtain a Be ground-state energy of -1.011704 a.u. in the absence of a laser field, which compares well with the experimental value -1.011850 a.u. obtained by Bashkin and Stoner [65]. Our results for the singly excited states given in Table II are compared with those of Bachau et al. [64] and Bashkin and Stoner [65]. Our results display three-digit agreement with the experimental results of Ref. [65], whereas they display only two-digit agreement with the theoretical results of Ref. [64]. We note that our calculations included the polarizability $\alpha_{c}$ of the $\mathrm{Be}^{2+}$ core in our potential $V(r)$ in Eq. (1), whereas those of Ref. [64] did not. The dynamic polarizability of the $\mathrm{Be}^{2+}$ core is not expected to play a significant role, since for the laser frequencies $\omega_{L}$ we employ, the dynamic polarizability of isoelectronic $\mathrm{He}$ is very close to its static one [66].

Our results for the doubly excited states are given in Table III, where they are compared with those of Refs. [54-56,58,59]. In nearly all cases shown, the agreement between our results and those of these other theoretical and experimental references is good to three digits. In Table III, we also list the widths $\Gamma$ of the autoionizing resonances obtained theoretically [54] or experimentally [58]. Our method for solving the time-independent Schrödinger equation uses real basis functions $R_{n l}(r)$ and hence cannot provide information about the widths $\Gamma$.

\section{B. Calculation of harmonic-generation spectra}

The numerical calculation of the harmonic generation spectrum is carried out as in Refs. [36,38], i.e., using the two-electron wave-packet solution of the two-electron TDSE. Since our numerical methods are described in detail in Sec. II B of Ref. [38], we only briefly summarize them here by noting the following four key points: (i) We treat the interaction between the atom and the laser field within the electric dipole approximation. (ii) Owing to gauge invariance, the HHG power spectrum can in principle be evaluated using the induced
TABLE III. Resonance energies $E_{d g} \equiv E_{d}-E_{g}$ (in $\mathrm{eV}$ ) and widths $\Gamma$ (in $\mathrm{eV}$ ) of ${ }^{1} P$ doubly excited autoionizing levels of Be converging to the $\mathrm{Be}^{+}(2 p), \mathrm{Be}^{+}(3 s), \mathrm{Be}^{+}(3 p)$, and $\mathrm{Be}^{+}(3 d)$ thresholds.

\begin{tabular}{|c|c|c|c|c|c|}
\hline \multirow[b]{2}{*}{ State } & \multicolumn{4}{|c|}{ (a) $E_{d g}(\mathrm{eV})$} & \multirow{2}{*}{$\frac{\text { (b) } \Gamma(\mathrm{meV})}{\text { Ref. [58] }}$} \\
\hline & This work & Ref. [54] & Ref. [55] & Ref. [58] & \\
\hline $2 p 3 s$ & 10.7120 & 10.9103 & 10.7068 & 10.8890 & 531.0 \\
\hline $2 p 4 s$ & 12.1425 & 12.0918 & 11.9678 & 12.1120 & 174.0 \\
\hline $2 p 5 s$ & 12.4934 & 12.5579 & 12.5339 & 12.5710 & 77.00 \\
\hline $2 p 6 s$ & 12.8063 & 12.7911 & 12.7820 & 12.8120 & 47.00 \\
\hline $2 p 7 s$ & 12.9067 & 12.9239 & 12.9219 & 12.9440 & 29.00 \\
\hline $2 p 8 s$ & 12.9731 & 13.0070 & 13.0100 & 13.0220 & 16.00 \\
\hline \multirow[t]{2}{*}{$2 p 9 s$} & 13.1019 & 13.0623 & & 13.0780 & 3.000 \\
\hline & & & & & Ref. [54] \\
\hline $2 p 3 d$ & 11.8325 & 11.8310 & 11.8623 & 11.8400 & 1 \\
\hline $2 p 4 d$ & 12.4409 & 12.4374 & 12.4658 & 12.4600 & 1 \\
\hline \multirow[t]{2}{*}{$2 p 5 d$} & 12.7294 & 12.7272 & 12.7570 & 12.7420 & 0.9 \\
\hline & & & & Ref. [56] & Ref. [54] \\
\hline $3 s 3 p$ & 17.6370 & 17.6640 & 17.6441 & 17.6844 & 507.4 \\
\hline $3 s 4 p$ & 18.8428 & 18.8548 & 18.7970 & 18.8342 & 75.10 \\
\hline $3 s 5 p$ & 19.6757 & 19.6524 & & & 51.20 \\
\hline $3 s 6 p$ & 19.8053 & 19.8119 & 19.8059 & 19.7965 & 55.90 \\
\hline $3 s 7 p$ & 19.9362 & 19.9117 & 19.9141 & 19.9141 & 51.10 \\
\hline \multirow[t]{2}{*}{$3 s 8 p$} & 20.0613 & 19.9828 & & 19.9944 & 34.80 \\
\hline & & & Ref. [59] & & Ref. [54] \\
\hline $3 p 4 s$ & 19.7013 & 19.6687 & & & 5.600 \\
\hline $3 p 5 s$ & 20.5111 & 20.4990 & 20.5000 & & 240.7 \\
\hline $3 p 6 s$ & 20.7915 & 20.7951 & 20.8000 & & 6.000 \\
\hline $3 p 7 s$ & 20.9211 & 20.9254 & & & 5.900 \\
\hline \multirow[t]{2}{*}{$3 p 8 s$} & 21.0090 & 21.0163 & & & 15.60 \\
\hline & & & Ref. [59] & & Ref. [54] \\
\hline $3 p 3 d$ & 19.3830 & 19.3919 & & & 4.500 \\
\hline $3 p 4 d$ & 20.3581 & 20.3556 & 20.3600 & & 5.600 \\
\hline \multirow[t]{2}{*}{$3 p 5 d$} & 20.7022 & 20.6963 & & & 3.800 \\
\hline & & & & & Ref. [54] \\
\hline $3 d 4 p$ & 20.1412 & 20.1120 & & & 5.300 \\
\hline $3 d 5 p$ & 20.5967 & 20.6070 & & & 185.6 \\
\hline \multirow[t]{2}{*}{$3 d 6 p$} & 20.9211 & 20.9317 & & & 17.10 \\
\hline & & & & & Ref. [54] \\
\hline $3 d 3 f$ & 20.2891 & 20.1980 & & & 6.200 \\
\hline $3 d 4 f$ & 20.7022 & 20.7268 & & & 6.300 \\
\hline $3 d 5 f$ & 20.9913 & 20.9877 & & & 5.400 \\
\hline
\end{tabular}

dipole in the length, velocity, or acceleration gauge. However, use of either the length gauge or the acceleration gauge requires accurate two-electron wave packets at either large or small radial coordinates, respectively, whereas the velocity gauge requires accurate wave packets at intermediate radial coordinates. For this reason we employ the dipole velocity gauge to evaluate the harmonic-generation spectrum. (iii) $\mathrm{We}$ employ a moderate laser peak intensity of $8-15 \mathrm{TW} / \mathrm{cm}^{2}$. (iv) Owing to the low energies of excited states in the field-free energy spectrum of the $\mathrm{Be}$ atom, we are able to carry out our calculations for a range of driving laser frequencies within which lies the fundamental of the tunable Ti:sapphire laser. With these choices of laser parameters, 
we are in the multiphoton regime and the ponderomotive energy and the quiver amplitude never exceed $0.7 \mathrm{eV}$ and 6.0 a.u., respectively. We find that calculating the induced dipole in the velocity gauge within a box of radius $r_{0}=$ 75 a.u. gives converged results for the HHG spectrum of Be.

The TDSE equation describing the interaction of the $\mathrm{Be}$ atom with a laser field in the dipole velocity gauge is

$$
i \frac{\partial}{\partial t} \Psi_{V}\left(\mathbf{r}_{1}, \mathbf{r}_{2}, t\right)=\left[H+\mathbf{A}(t) \cdot\left(\mathbf{p}_{1}+\mathbf{p}_{2}\right)\right] \Psi_{V}\left(\mathbf{r}_{1}, \mathbf{r}_{2}, t\right) .
$$

We parametrize the driving laser by its vector potential and electric field, polarized along the $z$ axis, and defined in the interval $-T / 2 \leqslant t \leqslant T / 2$ as

$$
\mathbf{A}(t)=A_{0} f(t) \sin \left(\omega_{L} t+\phi\right) \mathbf{e}_{z}, \quad \mathbf{E}(t)=-\frac{\partial}{\partial t} \mathbf{A}(t),
$$

where $\omega_{L}$ is the driving frequency, $\phi$ is the so-called carrierenvelope phase (CEP), and $T$ is the total pulse duration. The peak intensity of the laser field is given by $I=E_{0}^{2}$, where $E_{0}=A_{0} \omega_{L}$. Owing to the large number of optical cycles, $n_{c}$, in our laser pulse, the CEP effects are negligible. Note that the initial state of the time propagation is the field-free ground state $1 s^{2} 2 s^{2}\left({ }^{1} S\right)$ of Be and the norm of the wave packet, for the laser parameters employed here, is always well conserved. Once the time-dependent wave functions are obtained, the expectation value of the dipole velocity operator $d_{V}(t)$ is obtained as in Ref. [38], i.e., either in the original basis

$$
d_{V}(t)=\left\langle\Psi_{V}\left(\mathbf{r}_{1}, \mathbf{r}_{2}, t\right)\left|-i \frac{\partial}{\partial z_{1}}-i \frac{\partial}{\partial z_{2}}\right| \Psi_{V}\left(\mathbf{r}_{1}, \mathbf{r}_{2}, t\right)\right\rangle,
$$

or in the eigenstate basis. The power $\mathcal{P}\left(\omega_{L} ; \omega\right)$ of highharmonic radiation with frequency $\omega$ induced by the laser field with frequency $\omega_{L}$ is

$$
\mathcal{P}\left(\omega_{L} ; \omega\right)=|d(\omega)|^{2}, \quad d(\omega)=\frac{i}{\omega T} \int_{-T}^{T} d t e^{-i \omega t} d_{V}(t),
$$

where $d(\omega)$ is the Fourier transform of $d_{V}(t)$.

Toma et al. [67] found experimentally that resonance effects in HHG that may not be visible for a Gaussian-shaped focus can be observed for a spatially shaped flat top focus. We model such a focus in our calculations by a trapezoidal laser-pulse profile $f(t)$ (as in Refs. $[38,68,69]$ ) that is ramped on and off over $n_{c}^{s}$ optical cycles and has a total duration of $n_{c}$ optical cycles. In Fig. 1 we show the temporal evolution of the dipole velocity amplitude, $d_{V}(t)$ [cf. Eq. (8)], for a driving laser frequency $\omega_{L}=1.7346 \mathrm{eV}$ [which puts the seventh harmonic in resonance with the $2 p 4 s\left({ }^{1} P\right)$ doubly excited state], an intensity $I=10 \mathrm{TW} / \mathrm{cm}^{2}$, and $n_{c}^{s}=8$ optical cycles with a total of $n_{c}=30$ optical cycles. One sees that $d_{V}(t)$ oscillates with a period $2 \pi / \omega_{L}=2.4$ fs and mimics the flap-top profile of the pulse. It is in phase with the vector potential and out of phase by $\pi / 2$ with the electric field.

\section{HARMONIC-GENERATION RESULTS}

In this section we present our numerical results, which focus on the influence of the $2 p 4 s\left({ }^{1} P\right)$ and $2 p 5 s\left({ }^{1} P\right)$ autoionizing states on the Be HHG spectrum for driving laser frequencies in

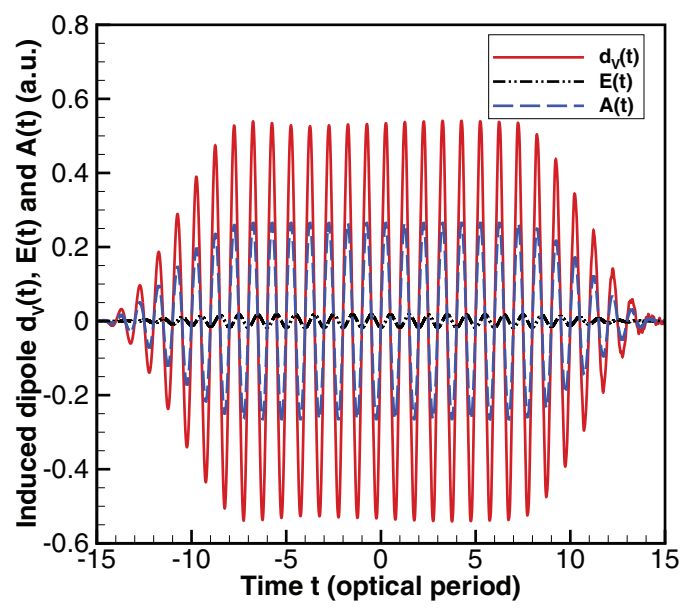

FIG. 1. (Color online) Time dependence of the induced dipole velocity amplitude $d_{V}(t)$, the electric field $E(t)$, and the vector potential $A(t)$ for a 30-cycle flat-top driving laser pulse with frequency $\omega_{L}=1.7346 \mathrm{eV}$ and peak intensity $I=10 \mathrm{TW} / \mathrm{cm}^{2}$.

the vicinity of seven-photon resonances with these two doubly excited states. In Sec. III A we note some key properties of these two resonances. In Sec. III B we show the behavior of low-order harmonics as a function of driving laser frequency in the vicinity of seven-photon resonance with these two doubly excited states. In Sec. IIIC we examine the highorder harmonic spectrum and predict a remarkably broad multiphoton-regime plateau in the HHG spectrum that forms when the doubly excited states are in seven-photon resonance with the driving laser frequency. In Secs. III D, III E, and III F, we focus on the integrated harmonic power for the resonant seventh harmonic and its dependence respectively on the driving laser frequency $\omega_{L}$, the pulse duration, and the peak pulse intensity.

\section{A. Positions, widths, and lifetimes of the $2 p 4 s\left({ }^{1} P\right)$ and $2 p 5 s\left({ }^{1} P\right)$ doubly excited states of $\mathrm{Be}$}

The odd-parity $2 p 4 s\left({ }^{1} P\right)$ and $2 p 5 s\left({ }^{1} P\right)$ doubly excited states (labeled for simplicity respectively by " $a$ " and " $b$ ") are located at excitation energies $E_{d g}^{a}=12.1425 \mathrm{eV}$ and $E_{d g}^{b}=$ $12.4934 \mathrm{eV}$ above the ground state. These excitation energies are in good agreement with the corresponding experimental values of $12.1120 \pm 0.174 \mathrm{eV}[58]$ and $12.5339 \pm 0.077 \mathrm{eV}$ [55]. These doubly excited states have widths of $\Gamma^{a}=$ $0.174 \mathrm{eV}$ and $\Gamma^{b}=0.077 \mathrm{eV}$, which are respectively five and two times larger than that of the He $2 s 2 p\left({ }^{1} P\right)$ doubly excited state, whose effects on the HHG spectrum of He were analyzed in Ref. [38]. These two states are the second and third doubly excited states in the Be $2 p n s\left({ }^{1} P\right)$ series (cf. Table III). We do not present results for the first doubly excited state in that series, i.e., the $2 p 3 s\left({ }^{1} P\right)$ state, owing to its very broad width $(\Gamma=0.531 \mathrm{eV}$ [58]). Also, although the $2 p n d\left({ }^{1} P\right)$ autoionizing states with $n=3,4$, and 5 are located at excitation energies in the vicinity of the $2 p 4 s\left({ }^{1} P\right)$ and $2 p 5 s\left({ }^{1} P\right)$ doubly excited states (cf. Table III), their widths of $\lesssim 1 \mathrm{meV}$ are so narrow that an extremely fine frequency mesh would be required to observe their effects. Moreover, they do not contribute significantly to the integrated harmonic 
power for the seventh harmonic of the Be spectrum that we investigate below.

In order for a seven-photon transition from the ground state to be in resonance with either the $2 p 4 s\left({ }^{1} \mathrm{P}\right)$ or the $2 p 5 s\left({ }^{1} \mathrm{P}\right)$ doubly excited state, the driving laser frequency should have the values, respectively, $\omega_{a}^{\text {res }}=E_{d g}^{a} / 7=1.7346 \mathrm{eV}$ or $\omega_{b}^{\text {res }}=E_{d g}^{b} / 7=1.7848 \mathrm{eV}$. Each of these estimates, of course, is based on the field-free energies; it is not known whether or by how much the resonance positions shift in the presence of the driving laser field. We note, however, that each of these estimated energies lies within the tunable range of the fundamental of the Ti:sapphire laser. In Fig. 2 we present a schematic diagram of the field-free singly and doubly excited states of Be with the excitation energies above the ground state and the lowest $\mathrm{Be}^{+}$ionization thresholds clearly indicated up to the energy of the double-ionization threshold. The upward-pointing arrows indicate a seven-photon resonant transition (with driving frequency $\approx 1.7346 \mathrm{eV}$ ) from the ground state to the $2 p 4 s\left({ }^{1} P\right)$ doubly excited state followed

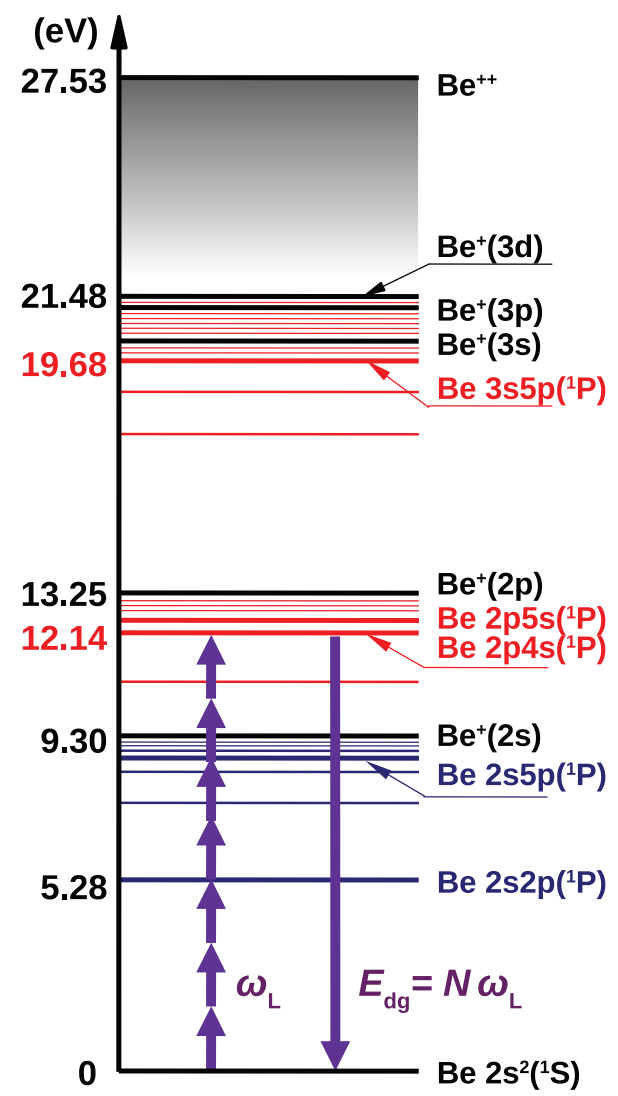

FIG. 2. (Color online) Field-free singly excited (blue lines) and doubly excited (red lines) states of Be plotted vs excitation energy above the ground state. The gray region above the $\mathrm{Be}^{+}$ $3 d$ excitation threshold indicates higher doubly excited states and excitation thresholds converging to the double-ionization threshold. The (purple) arrows indicate an $(N=7)$-photon transition (with driving frequency $\omega_{L} \approx 1.734 \mathrm{eV}$ ) from the ground state to the $2 p 4 s\left({ }^{1} P\right)$ doubly excited state followed by stimulated emission of a harmonic photon back to the ground state. Note that three- and five-photon transitions from the ground state are close to resonance respectively with the $2 s 2 p\left({ }^{1} P\right)$ and/or $2 s 5 p\left({ }^{1} P\right)$ singly excited states.
TABLE IV. Laser frequencies $\omega_{a, b}^{\mathrm{res}}$ in seven-photon resonance with the (a) $2 p 4 s\left({ }^{1} \mathrm{P}\right)$ and (b) $2 p 5 s\left({ }^{1} \mathrm{P}\right)$ autoionizing states of $\mathrm{Be}$ (where $\omega_{a, b}^{\mathrm{res}}=E_{d g}^{a, b} / 7, E_{d g}^{a}=12.1425 \mathrm{eV}$, and $E_{d g}^{b}=12.4934 \mathrm{eV}$ ) and their harmonics, $\Omega_{q} \equiv q \omega_{a, b}^{\mathrm{res}}$ for $1 \leqslant q \leqslant 17$.

\begin{tabular}{lcc}
\hline \hline \multicolumn{3}{c}{ Harmonic energy $\Omega_{q} \equiv q \omega_{a, b}^{\text {res }}(\mathrm{eV})^{\mathrm{a}}$} \\
\hline$q$ & $2 p 4 s\left({ }^{1} P\right)$ & $2 p 5 s\left({ }^{1} P\right)$ \\
\hline 1 & $q \omega_{a}^{\text {res }}$ & $q \omega_{b}^{\text {res }}$ \\
3 & 1.73464 & 1.7848 \\
5 & $\mathbf{5 . 2 0 3 9 3}$ & 5.3543 \\
7 & $\mathbf{8 . 6 7 3 2 1}$ & $\mathbf{8 . 9 2 3 8}$ \\
9 & $\mathbf{1 2 . 1 4 2 5} *$ & $\mathbf{1 2 . 4 9 3 4}$ \\
11 & 15.61179 & 16.0629 \\
13 & 19.08107 & $\mathbf{1 9 . 6 3 2 4}$ \\
15 & 22.55036 & 23.2020 \\
17 & 26.01964 & 26.7715 \\
\hline \hline
\end{tabular}

${ }^{a}$ Harmonic energies in bold are close to singly or doubly excited state excitation energies (cf. Tables II and III); the asterisks highlight the resonant harmonic order $q=7$.

by stimulated harmonic emission back to the ground state, indicated by the single downward arrow. By slightly varying $\omega_{L}$ to $\omega_{L} \approx 1.7848 \mathrm{eV}$, a seven-photon resonant transition can reach the $2 p 5 s\left({ }^{1} P\right)$ autoionizing state from the ground state.

In the presence of the laser field, the excitation energies of the singly and doubly excited states may be shifted. Therefore, in this paper we vary the frequency $\omega_{L}$ over a small energy range in order to ensure achievement of seven-photon resonances with the $2 p 4 s\left({ }^{1} P\right)$ and $2 p 5 s\left({ }^{1} P\right)$ doubly excited states. As $\omega_{L}$ varies, some low-order transitions become close to resonance with transitions between the ground state and some singly excited states of Be. For later reference, we highlight in bold font in Table IV those harmonics of the two driving laser frequencies in seven-photon resonance with the two doubly excited states that are also close to resonance with some singly excited states (see Table II and Fig. 2) and some other doubly excited states (see Table III and Fig. 2).

In Table $\mathrm{V}$ we compare the lifetimes of each of these doubly excited states with the optical period of the driving laser field at the estimated resonance frequencies. One sees that the lifetimes of these autoionizing states are very short: each has a lifetime equal to only a few optical periods of the driving laser field, i.e., $\tau^{a}=1.6 T_{0}^{a}$ and $\tau^{b}=3.7 T_{0}^{b}$. A key question addressed in this work is whether such short-lived doubly excited states can have an influence on the HHG spectrum of Be.

TABLE V. Widths, $\Gamma^{a, b}$ [58], and lifetimes, $\tau^{a, b} \equiv 1 / \Gamma^{a, b}[60]$, of the (a) $2 p 4 s\left({ }^{1} P\right)$ and (b) $2 p 5 s\left({ }^{1} P\right)$ doubly excited states of Be. For the field-free resonant driving frequencies $\omega_{a}^{\text {res }}=1.7346 \mathrm{eV}$ and $\omega_{b}^{\mathrm{res}}=1.7848 \mathrm{eV}$, the corresponding optical periods, $T_{0}^{a, b} \equiv 2 \pi / \omega_{a, b}^{\mathrm{res}}$, are also given.

\begin{tabular}{lccccc}
\hline \hline$\Gamma^{a}(\mathrm{eV})$ & $\tau^{a}(\mathrm{fs})$ & $T_{0}^{a}(\mathrm{fs})$ & $\Gamma^{b}(\mathrm{eV})$ & $\tau^{b}(\mathrm{fs})$ & $T_{0}^{b}(\mathrm{fs})$ \\
\hline 0.174 & 3.78 & 2.38 & 0.077 & 8.55 & 2.32 \\
\hline \hline
\end{tabular}



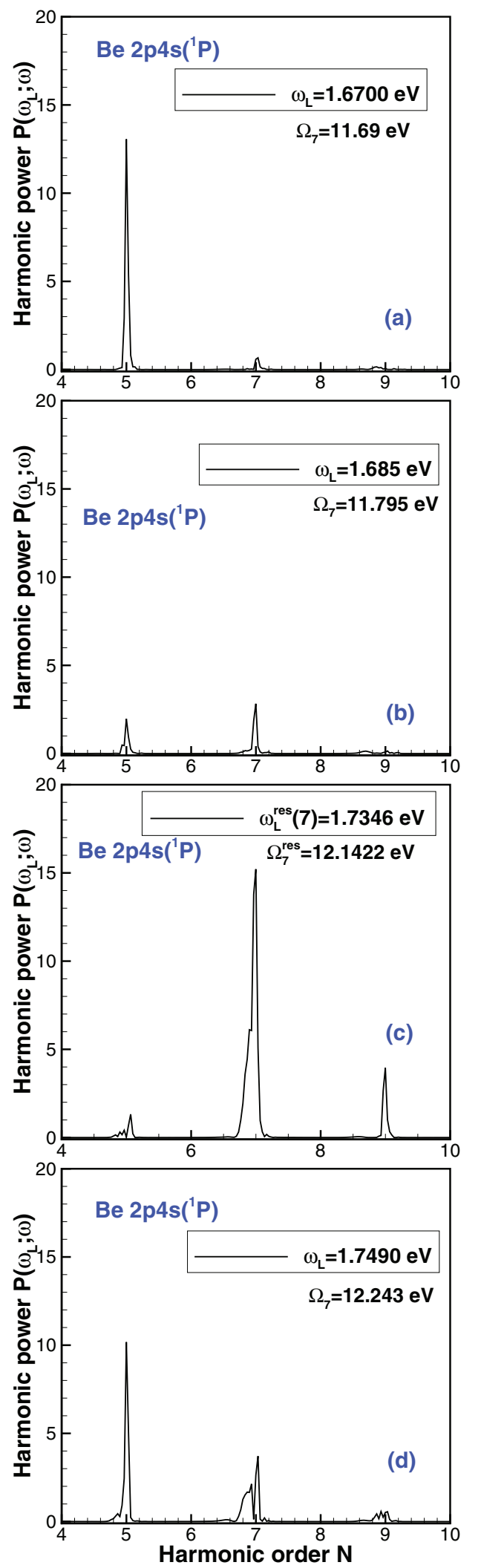

FIG. 3. (Color online) Harmonic power $\mathcal{P}\left(\omega_{L} ; \omega\right)$ (a.u.) [cf. Eq. (9)] for the seventh harmonic (and its two nearest neighbors) for four driving laser frequencies $\omega_{L}$ that put the seventh harmonic frequency $\Omega_{7} \equiv 7 \omega_{L}$ below, above, and in resonance with the Be $2 p 4 s\left({ }^{1} P\right)$ autoionizing state. The harmonic order is $N \equiv \omega / \omega_{L}$ and the laser-pulse peak intensity is $10 \mathrm{TW} / \mathrm{cm}^{2}$.
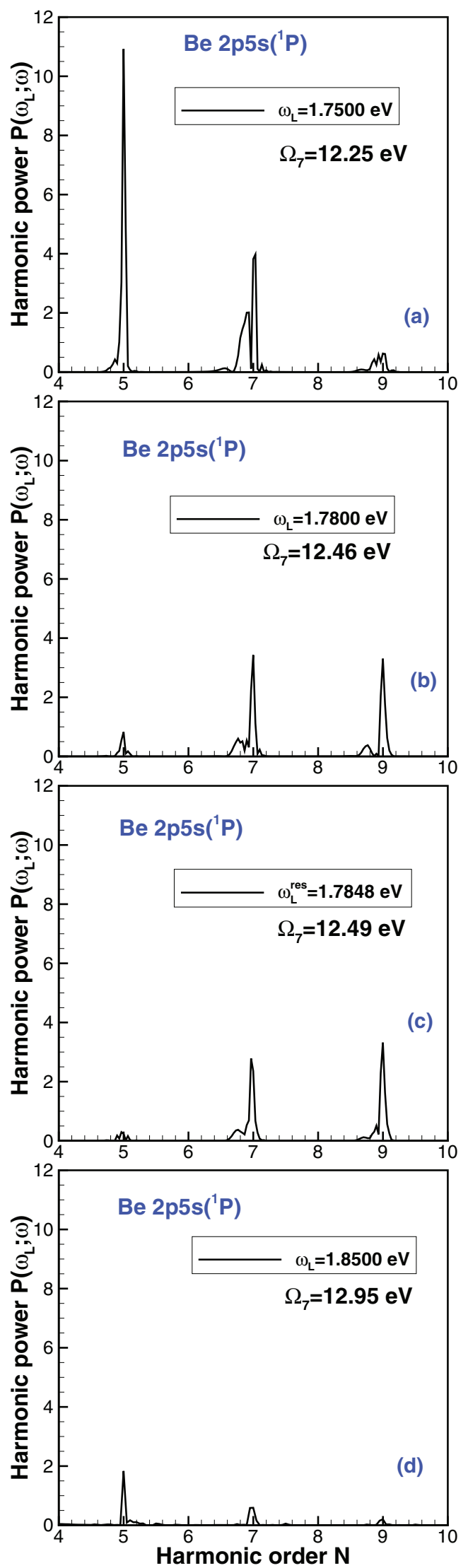

FIG. 4. (Color online) Same as in Fig. 3 but for frequencies $\omega_{L}$ below, above, and in resonance with the $\mathrm{Be} 2 p 5 s\left({ }^{1} P\right)$ doubly excited state. 


\section{B. Driving laser frequency dependence of the fifth, seventh, and ninth harmonics}

For values of $\omega_{L}$ in the neighborhood of the two resonant frequencies of $\omega_{a}^{\text {res }}=1.7346 \mathrm{eV}$ and $\omega_{b}^{\text {res }}=1.7848 \mathrm{eV}$ and for a moderate laser peak intensity of $10 \mathrm{TW} / \mathrm{cm}^{2}$, the corresponding ponderomotive energies, $U_{p}$, are about $0.5 \mathrm{eV}$. The corresponding Keldysh parameters, $\gamma$, are about 3, thus indicating that the HHG spectra are produced in the multiphoton regime, in which perturbation theory is valid except near resonant frequencies. For nonresonant driving laser frequencies, we thus expect the intensities of successive harmonics to decrease with harmonic order.

This is indeed the case in Figs. 3(a) and 3(d), and Figs. 4(a) and 4(d) for which $\omega_{L}$ is respectively below and above the resonant laser frequency $\omega_{a}^{\text {res }}$ or $\omega_{b}^{\text {res }}$. But when $\omega_{L}$ is close to or on resonance with the $2 p 4 s\left({ }^{1} P\right)$ autoionizing state, the harmonic power becomes much greater than that of its nearest neighbors [see Figs. 3(b) and 3(c)]. Moreover, we note in both cases the diminution of the intensity of the fifth harmonic when $\omega_{L} \approx \omega_{a, b}^{\text {res }}$ indicating that the fifth harmonic feeds the seventh harmonic. Furthermore, in Figs. 4(b) and 4(c), the seventh and the ninth harmonics have comparable intensities indicating additional strong effects which act to lower the intensity of the resonant seventh harmonic and to increase that of the nonresonant ninth harmonic.

\section{Plateau structure in the multiphoton regime HHG spectrum of Be}

In Fig. 5 we present our results for the HHG spectrum of Be up to the 29th harmonic for the laser parameters given in Fig. 1. Four curves are given, differing only in the number (from 7 to 10) of total angular momentum values $L$ included in our calculations. One sees that our results are converged. One notices also that the peak harmonic powers display a converged plateau structure with a cutoff located at about harmonic order $N \approx 25$. In the tunneling regime, the well-known HHG plateau structure originates from rescattering effects and the cutoff

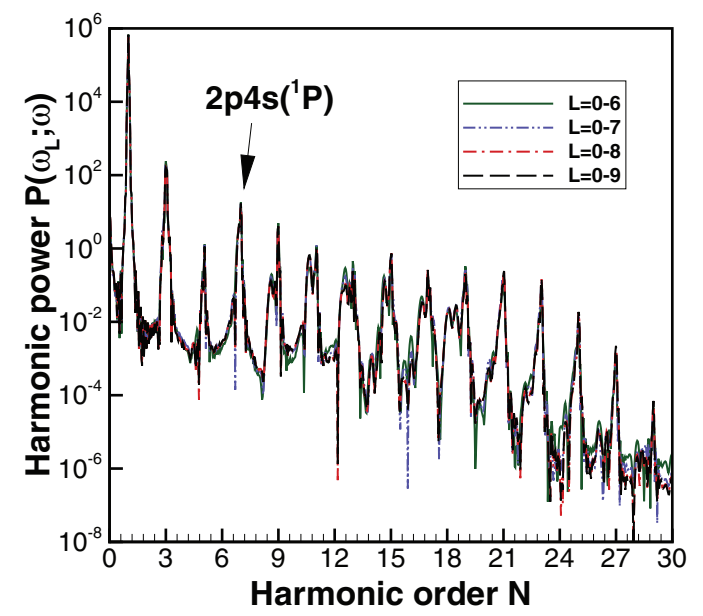

FIG. 5. (Color online) Convergence of the HHG spectrum of Be [cf. Eq. (9)] as a function of the total angular momentum $L$ by a 30 -cycle flat-top shaped laser for frequency $\omega_{L}=1.7346 \mathrm{eV}$ [which puts the seventh harmonic on resonance with the $2 p 4 s\left({ }^{1} P\right)$ doubly excited state]. The peak pulse intensity is $10 \mathrm{TW} / \mathrm{cm}^{2}$.
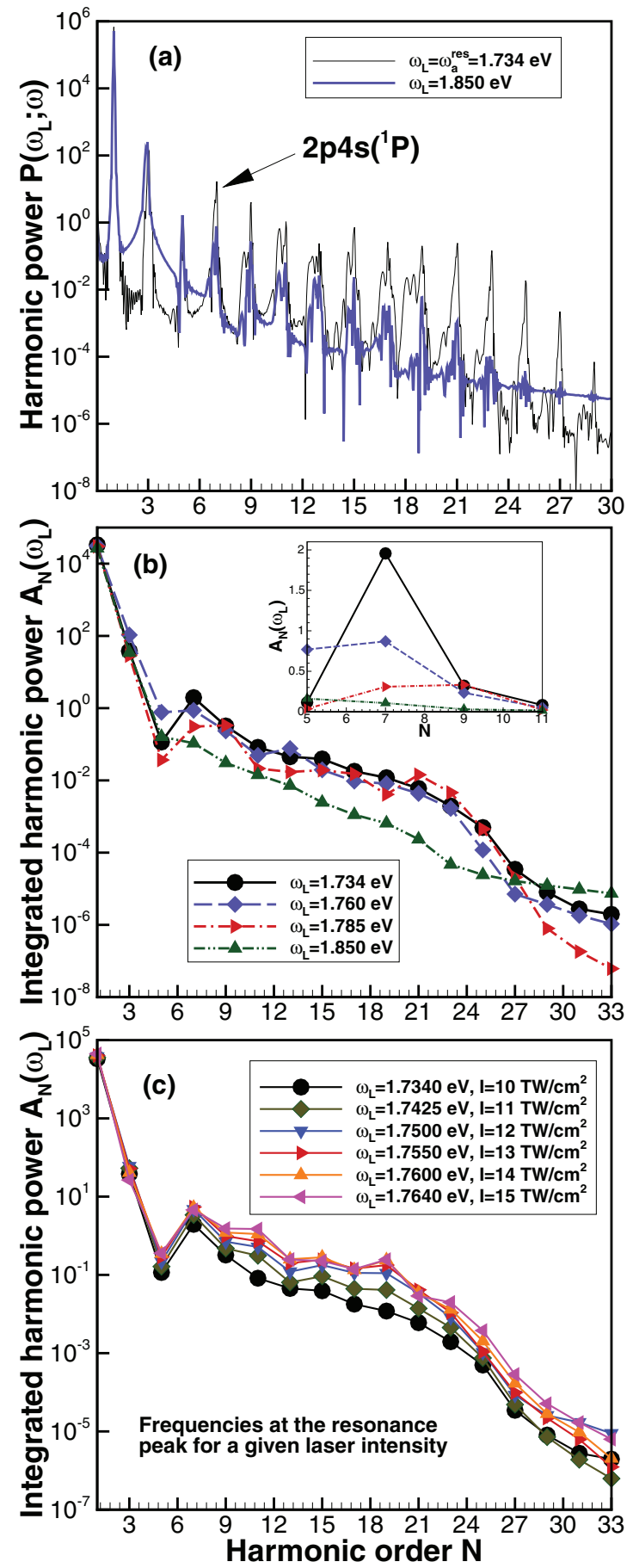

FIG. 6. (Color online) Plateau structure in the HHG spectrum of Be. (a) Harmonic power spectra [cf. Eq. (9)] for two driving laser frequencies: $\omega_{L} \approx \omega_{a}^{\text {res }}=1.734 \mathrm{eV}$ [which puts the seventh harmonic on resonance with the $2 p 4 s\left({ }^{1} P\right)$ doubly excited state] and $\omega_{L}=1.85 \mathrm{eV}$ (which is off resonance). (b) Spectra of integrated harmonic powers $A_{N}\left(\omega_{L}\right)$ [cf. Eq. (10)] for four different driving laser frequencies: the two as in (a) plus $\omega_{L}=1.76 \mathrm{eV}$ [which puts the third harmonic close to resonance with the $2 s 2 p\left({ }^{1} P\right)$ singly excited state] and $\omega_{L} \approx \omega_{b}^{\text {res }}=1.785 \mathrm{eV}$ [which puts the seventh harmonic on resonance with the $2 p 5 s\left({ }^{1} P\right)$ doubly excited state]. The inset graph shows the lowest integrated harmonic powers on a linear scale. In (a) and (b), the peak pulse intensity is $10 \mathrm{TW} / \mathrm{cm}^{2}$. (c) Intensity dependence of the plateau structure for laser frequencies taken at the peak of the seventh harmonic resonance (see Fig. 12). 
occurs at the odd harmonic order $N$ closest to $I_{0}+3.17 U_{p}$, where $I_{0}$ is the one-electron binding energy of the system and $U_{p}$ is the ponderomotive potential. In the present case, however, for the laser parameters in Fig. 1 we are in the multiphoton regime with $I_{0}=9.3 \mathrm{eV}$ and $U_{p}=0.48 \mathrm{eV}$. The tunneling regime cutoff prediction of the three-step model for these parameters would be at $N=5$ or 7 . We note also that plateau structures in the multiphoton regime have been predicted by Frolov et al. [70] for the $F^{-}$ion with the same cutoff as predicted by the three-step model. However, Ref. [70] noted that such plateaus only appear for $U_{p} \geqslant \omega_{L}$. In our case, however, $U_{p}=0.275 \omega_{L}$, so that although we are in the multiphoton regime, the parameters of the present problem do not meet the criteria given in Ref. [70] for a multiphoton regime plateau in the HHG spectrum. Finally, we emphasize that the tunneling regime three-step model and the model system described in Ref. [70] employ a single-active-electron approach and do not treat resonance effects, whereas in this work we treat the $\mathrm{Be}$ atom as a two-active-electron problem and focus on resonance effects.

In Fig. 6 we analyze the plateau structure in the Be HHG spectrum. In Fig. 6(a) we compare the harmonic power spectrum [see Eq. (9)] for the driving laser frequency $\omega_{L} \approx$ $\omega_{a}^{\text {res }}=1.734 \mathrm{eV}$ [on resonance with the field-free $2 p 4 s\left({ }^{1} P\right)$ doubly excited state] with that for the nonresonant frequency $\omega_{L}=1.85 \mathrm{eV}$. The effect of the seven-photon resonance with the doubly excited state is to raise the peaks of all harmonic powers $N \geqslant 7$.

In order to enhance the visibility of the multiphoton regime plateau, in Figs. 6(b) and 6(c) we plot $A_{N}\left(\omega_{L}\right)$ [38], the harmonic power integrated over the energies from $(N-1) \omega_{L}$ to $(N+1) \omega_{L}$, at each harmonic order $N$ :

$$
A_{N}\left(\omega_{L}\right)=\int_{(N-1) \omega_{L}}^{(N+1) \omega_{L}} \mathcal{P}\left(\omega_{L} ; \omega\right) d \omega .
$$

One sees in Fig. 6(b) that the plateau structure is now much more clearly visible, as is the position of the cutoff at $N \approx 25$. Results are given for four frequencies: $\omega_{a}^{\text {res }}=1.734 \mathrm{eV}, \omega_{L}=$ $1.76 \mathrm{eV}$ [which puts the third harmonic close to resonance with the $2 s 2 p\left({ }^{1} P\right)$ singly excited state], $\omega_{b}^{\text {res }}=1.785 \mathrm{eV}$, and $\omega_{L}=1.85 \mathrm{eV}$ (which is off resonance). The first three resonant frequencies all lead to a similarly shaped harmonic plateau, whereas the off-resonant frequency does not. Results for the lowest-order harmonics are shown in the inset graph in Fig. 6(b). One sees that the frequency $\omega_{L}=1.76 \mathrm{eV}$ that is close to resonance with the $2 s 2 p\left({ }^{1} P\right)$ singly excited state (cf. Table II) greatly increases the magnitude of the integrated harmonic power of the seventh harmonic. Since $7 \times 1.76 \mathrm{eV}=12.32 \mathrm{eV}$, we note from Table III that this energy is within one width $\Gamma$ of the position of the $2 p 4 s\left({ }^{1} P\right)$ doubly excited state.

In Fig. 6(c) we show the intensity dependence of the multiphoton regime plateau structure. The results in Figs. 6(a) and 6(b) are all for a driving laser peak pulse intensity of $I=10^{13} \mathrm{~W} / \mathrm{cm}^{2}$. This intensity is so low that the resonance features appear at energies close to their field-free values. In Fig. 6(c) we plot the spectrum of integrated harmonic powers for six different peak pulse intensities and note that, as the intensity increases, the resonance positions also shift slightly.
Therefore, each of the curves is for a different frequency $\omega_{L}$ corresponding to the maximum integrated harmonic power for the seventh harmonic, as will be discussed in more detail in Sec. III F below. The values of these frequencies are indicated in the legend of Fig. 6(c). Not surprisingly, the magnitude of the plateau increases with increasing peak pulse intensity. However, the cutoff remains at $N \approx 25$.

The position of the cutoff of the predicted multiphoton regime plateau at $N \approx 25$ requires a dynamical explanation. Since the plateau only occurs when there is a seven-photon resonance with the $2 p 4 s\left({ }^{1} P\right)$ or $2 p 5 s\left({ }^{1} P\right)$ doubly excited states, we can consider the generation of the $N$ th harmonic to take place in three steps: (i) a seven-photon transition from the Be ground state to the $2 p 4 s\left({ }^{1} \mathrm{P}\right)$ [or $2 p 5 s\left({ }^{1} \mathrm{P}\right)$ ] doubly excited state; (ii) an $(N-7)$-photon transition from the $2 p 4 s\left({ }^{1} P\right)$ [or $2 p 5 s\left({ }^{1} P\right)$ ] doubly excited state to the continuum $p$ state with kinetic energy $k_{N}^{2} / 2=N \omega_{L}-I_{0}$ in the field of the ground state of the ion, $\mathrm{Be}^{+} 1 s^{2} 2 s$; and (iii) the emission of a (single) harmonic photon from that continuum $p$ state to the Be $1 s^{2} 2 s^{2}$ ground state. We note that if autoionization [i.e., $\left.2 p 4 s\left({ }^{1} P\right) \rightarrow 2 s \in p\left({ }^{1} P\right)\right]$ occurs after step (i), the continuum electron has a kinetic energy $\epsilon=2.85 \mathrm{eV}$; a classical orbit calculation for a free electron in the field of the laser with such an initial kinetic energy indicates that there are no solutions for which that electron returns to the $\mathrm{Be}^{+}$ion. Let us focus on step (ii), which involves an $(N-7)$-photon transition from the doubly excited state to a $p$-continuum electron in the field of the ground state of the $\mathrm{Be}^{+}$ion. This transition, despite having a quite complicated transition operator [involving $(N-7)$ electric dipole operators and $(N-8)$ Green's functions], will include the overlap of the radial wave functions of the doubly excited state and the singly excited continuum $p$-electron state. Ignoring the complicated transition operator, in Fig. 7 we plot the absolute square of the radial overlap integral $\left|\left\langle R_{n s} \mid \Phi_{k_{N} p}\right\rangle\right|^{2}$ for $n=4$ and 5 as a function of the harmonic order $N$. Remarkably, each of the two overlap integrals has a maximum for a continuum kinetic energy corresponding to the $N=27$ harmonic, although the values for $N=25$

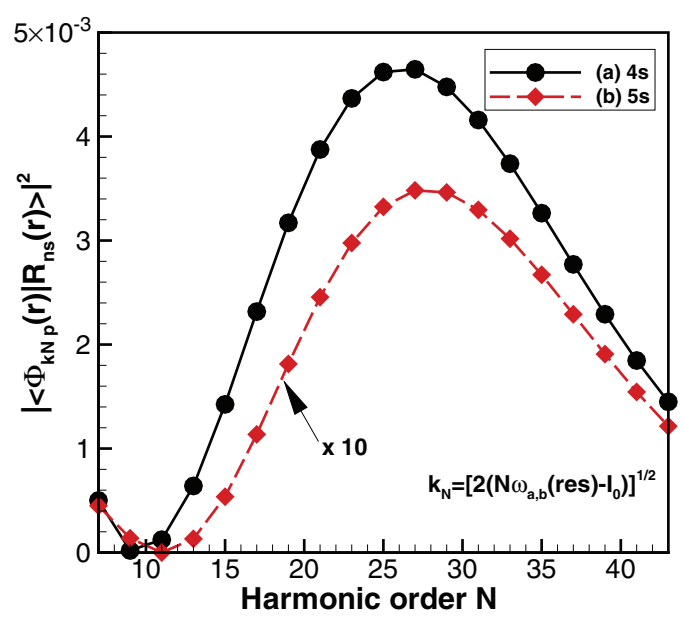

FIG. 7. (Color online) Absolute square of the overlap between the radial wave functions of the $\mathrm{Be} n s$ state (for $n=4,5$ ) and the $\mathrm{Be}^{+}$ continuum $p$ state with wave number $k_{N}$ corresponding to the kinetic energy of an electron after absorbing $N$ laser photons. 
are close to the maximum. One may thus speculate that this increase in the wave-function overlap as the harmonic order $N$ increases may compensate for the normally expected decrease of the harmonic transition amplitude, at least up to the value $N \approx 25$. While this admittedly very speculative explanation of the numerically predicted multiphoton regime plateau cutoff at $N \approx 25$ is certainly not definitive, it does indicate that a relevant component of a more complete explanation has its peak value at about that harmonic number. We note also that a similar overlap integral of the radial wave function of the continuum $p$ state and the $2 s$ bound state is monotonically decreasing with increasing harmonic order $N$. This latter fact may indicate that the three-photon resonance with the $2 s 2 p\left({ }^{1} P\right)$ singly excited state is not the cause of the plateau, but rather that the $2 s 2 p\left({ }^{1} P\right)$ resonance serves to "feed" the seven-photon resonance with the $2 p 4 s\left({ }^{1} P\right)$ doubly excited state [see the inset graph in Fig. 6(b)], which does produce the numerically predicted plateau.

\section{Driving laser frequency dependence of the integrated harmonic power}

In Figs. 8(a) and 8(b), the variation of the integrated harmonic power $A_{N}\left(\omega_{L}\right)$ for the third, fifth, and seventh harmonics as a function of $\omega_{L}$ are displayed for the same laser parameters as in Fig. 1. Owing to the relatively broad widths of the $2 p 4 s\left({ }^{1} P\right)$ and $2 p 5 s\left({ }^{1} P\right)$ doubly excited states (respectively $\Gamma^{a}=0.174 \mathrm{eV}$ and $\Gamma^{b}=0.077 \mathrm{eV}$ ), the laser photon energy ranges $\left(E_{d g}^{a, b} \pm \Gamma^{a, b}\right) / 7$ over which these two doubly excited states influence the seventh harmonic are respectively $1.71 \mathrm{eV} \leqslant \omega_{L} \leqslant 1.76 \mathrm{eV}$ and $1.77 \mathrm{eV} \leqslant \omega_{L} \leqslant$ $1.79 \mathrm{eV}$. However, instead of two maxima, the curve for the seventh harmonic [see Fig. 8(b)] exhibits three relative maxima within the photon energy range $1.71 \mathrm{eV} \leqslant \omega_{L} \leqslant 1.8 \mathrm{eV}$. The first maximum corresponds to seven-photon resonance with the $2 p 4 s\left({ }^{1} P\right)$ doubly excited state, and the third one to seven-photon resonance with the $2 p 5 s\left({ }^{1} P\right)$ doubly excited state. The middle peak stems from three-photon resonance with the $2 s 2 p\left({ }^{1} P\right)$ singly excited state: as shown in Fig. 8(a), this resonance greatly increases $A_{3}\left(\omega_{L}\right)$ as well as $A_{5}\left(\omega_{L}\right)$.

Also, Fig. 8(a) shows that the integrated third harmonic power, $A_{3}\left(\omega_{L}\right)$, has a shoulder structure at $\omega_{L} \approx \omega_{a}^{\text {res }}$ and a minimum at $\omega_{L} \approx \omega_{b}^{\text {res }}$, whereas the integrated fifth harmonic power, $A_{5}\left(\omega_{L}\right)$, has minima at both of these resonant driving frequencies. These minima may be interpreted as the feeding of the seventh harmonic by the fifth and third harmonics. The shoulder structure in $A_{3}\left(\omega_{L}\right)$ just above $\omega_{L}=1.73 \mathrm{eV}$ may stem from the combined effects of the $2 p 4 s\left({ }^{1} P\right)$ doubly excited state and the $2 s 5 p\left({ }^{1} P\right)$ singly excited state at these frequencies. It suffices to note that the third harmonic strongly interacts with higher harmonics, modifying their frequency dependence and increasing their intensities. Moreover, the five-photon resonant transition from the ground state to the $2 s 6 p\left({ }^{1} P\right)$ singly excited state (cf. Table II) is located just $0.07 \mathrm{eV}$ below the $2 p 4 s\left({ }^{1} P\right)$ doubly excited state and may also affect the intensity and spectral shape of the seventh harmonic.

Since it is only the seventh harmonic that is in seven-photon resonance with the $2 p 4 s\left({ }^{1} P\right)$ and $2 p 5 s\left({ }^{1} P\right)$ doubly excited states and not the fifth harmonic, by taking the ratio of the integrated harmonic powers, $A_{7}\left(\omega_{L}\right) / A_{5}\left(\omega_{L}\right)$ (which defines

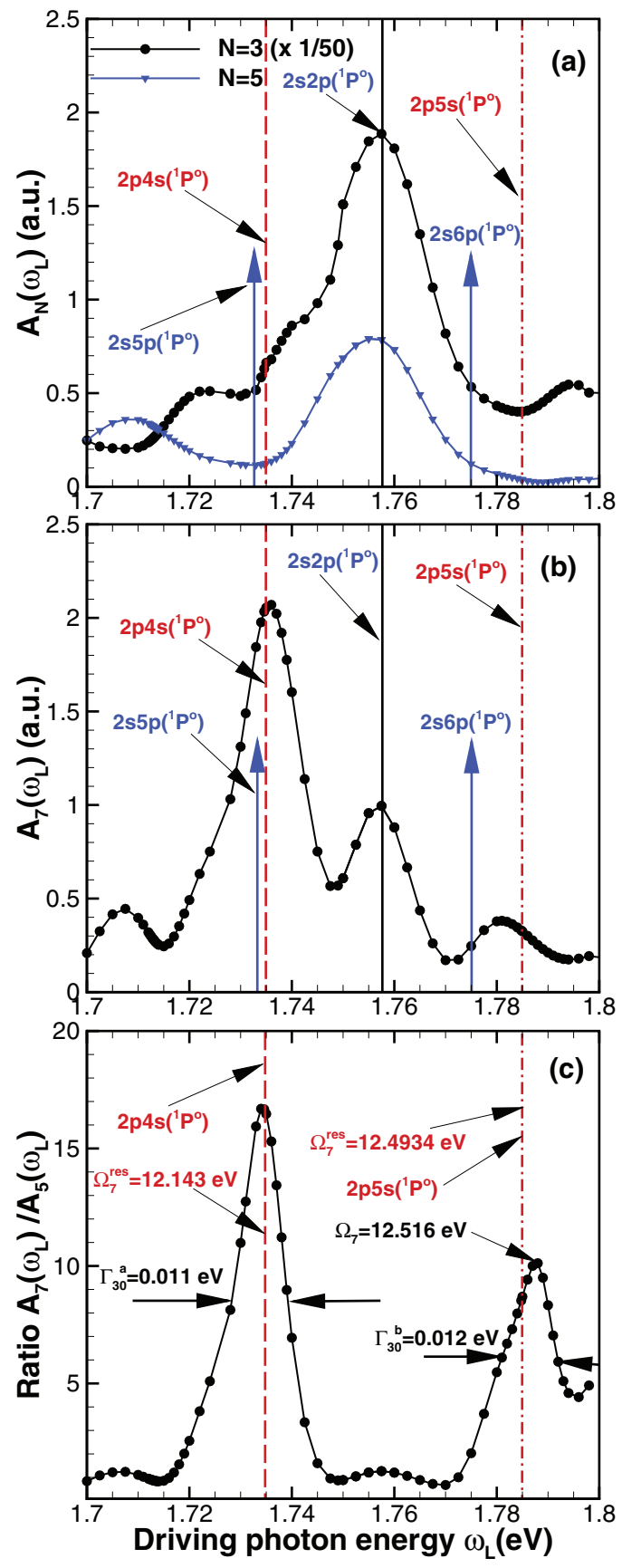

FIG. 8. (Color online) Variation of the integrated harmonic power $A_{N}\left(\omega_{L}\right)$ [Eq. (10)] with driving laser frequency $\omega_{L}$ and a peak pulse intensity of $10 \mathrm{TW} / \mathrm{cm}^{2}$. (a) $A_{3}\left(\omega_{L}\right)$ and $A_{5}\left(\omega_{L}\right)$; (b) $A_{7}\left(\omega_{L}\right)$; (c) the ratio $A_{7}\left(\omega_{L}\right) / A_{5}\left(\omega_{L}\right)$. The vertical dashed and dash-dotted lines mark $\omega_{a}^{\text {res }}=1.7848 \mathrm{eV}$ and $\omega_{b}^{\text {res }}=1.78477 \mathrm{eV}$, at which the seventh harmonic is resonant with the $2 p 4 s\left({ }^{1} \mathrm{P}\right)$ and $2 p 5 s\left({ }^{1} \mathrm{P}\right)$ doubly excited states, respectively. Solid lines in (a) and (b) mark $\omega_{L}$ at which the third harmonic is close to resonance with the $2 s 2 p\left({ }^{1} P\right)$ singly excited state. The blue arrows mark the five-photon resonant transitions to the $2 s 5 p\left({ }^{1} P\right)$ and $2 s 6 p\left({ }^{1} P\right)$ singly excited states. Note the scale factor in (a).

the enhancement of the seventh harmonic relative to the fifth harmonic), the frequency structure of this ratio may be regarded as originating predominantly from seven-photon resonances with the two autoionizing states, i.e., the influence 
of the lower-order resonance transitions to singly excited states is expected to be minor [38].

The frequency dependence of the ratio $A_{7}\left(\omega_{L}\right) / A_{5}\left(\omega_{L}\right)$ is displayed in Fig. 8(c). This ratio exhibits two large maxima with widths equal to $\Gamma_{30}^{a}=0.011 \mathrm{eV}$ (for the first resonance) and $\Gamma_{30}^{b}=0.012 \mathrm{eV}$ (for the second resonance). The first resonance located at $\omega_{L}=1.734 \mathrm{eV}\left(\Omega_{7} \equiv 7 \omega_{L}=12.138 \mathrm{eV}\right)$ is almost exactly (i.e., to four significant digits; cf. Table III) at the position of the field-free $2 p 4 s\left({ }^{1} P\right)$ doubly excited state. Its peak is symmetric and has a magnitude of about 16 . The second resonance has its maximum at $\omega_{L}=1.788 \mathrm{eV}$ $\left(\Omega_{7} \equiv 7 \omega_{L}=12.516 \mathrm{eV}\right)$, which is $0.0226 \mathrm{eV}$ above the field-free position of the $2 p 5 s\left({ }^{1} P\right)$ doubly excited state (cf. Table III). This second resonance peak is asymmetric and has a peak value of about 10 .

Finally, we note that the peak in $A_{7}\left(\omega_{L}\right)$ at the position of the $2 s 2 p\left({ }^{1} P\right)$ singly excited state near $\omega_{L} \approx 1.76 \mathrm{eV}$ [cf. Fig. 8(b)] becomes far less prominent in the spectral shape of the ratio $A_{7}\left(\omega_{L}\right) / A_{5}\left(\omega_{L}\right)$ [cf. Fig. 8(c)]. Similarly, the small peak just below $\omega_{L}=1.71 \mathrm{eV}$ in Fig. 8(b) also becomes far less prominent in the ratio shown in Fig. 8(c). Taking such integrated harmonic power ratios is thus an effective means to isolate the effects of doubly excited state resonances from those stemming from singly excited state resonances.

\section{E. Driving laser-pulse duration effects on the ratio of integrated harmonic powers $A_{7}\left(\omega_{L}\right) / A_{5}\left(\omega_{L}\right)$}

An important result in Sec. IIID is that the resonance features of the $2 p 4 s\left({ }^{1} P\right)$ and $2 p 5 s\left({ }^{1} P\right)$ doubly excited states on the ratio of integrated harmonic powers, $A_{7}\left(\omega_{L}\right) / A_{5}\left(\omega_{L}\right)$, have quite small numerical widths for the case of our 30-cycle, flat-top driving laser pulse. As shown in Fig. 8(c), $\Gamma_{30}^{a}=$ $0.011 \mathrm{eV}$ and $\Gamma_{30}^{b}=0.012 \mathrm{eV}$. The widths of these resonance features are thus an order of magnitude smaller than the measured and calculated widths of these autoionizing resonances (see Table I of Ref. [58]): $\Gamma^{a}=174 \pm 10 \mathrm{meV}$ and $\Gamma^{b}=77 \pm 10 \mathrm{meV}$, respectively. To understand these findings, it is necessary to investigate the dependence of these two resonance features in our calculated harmonic spectra on the temporal duration of the driving laser pulse.

We note first that no doubly excited state resonance features were found in HHG spectra produced by driving laser field pulses having durations shorter than the lifetimes of the doubly excited states. Thus, from Table $\mathrm{V}$, we see that in order to observe resonance with the $2 p 4 s\left({ }^{1} \mathrm{P}\right)$ or $2 p 5 s\left({ }^{1} \mathrm{P}\right)$ doubly excited states in the integrated harmonic power of the seventh harmonic, the laser-pulse duration must be $\gtrsim 2$ or $\gtrsim 4$ optical cycles, respectively. In fact, in our calculations we have found that evidence of these autoionizing states in the spectrum of the seventh harmonic is very weak for pulse durations $\lesssim 10$ optical cycles. These results for Be HHG spectra are consistent with our previous results for He [38], in which we predicted resonance features in the ninth, 11th, and 13th harmonics for a driving laser with frequency in the range $4.6 \mathrm{eV} \leqslant \omega_{L} \leqslant 6.6 \mathrm{eV}$ due to resonance with the $\mathrm{He} 2 s 2 p\left({ }^{1} P\right)$ doubly excited state. Owing to the relatively long lifetime $(\tau=1 / \Gamma=17.7 \mathrm{fs}$ ) of that doubly excited state, driving laser pulses with durations of about 30 optical cycles were required.

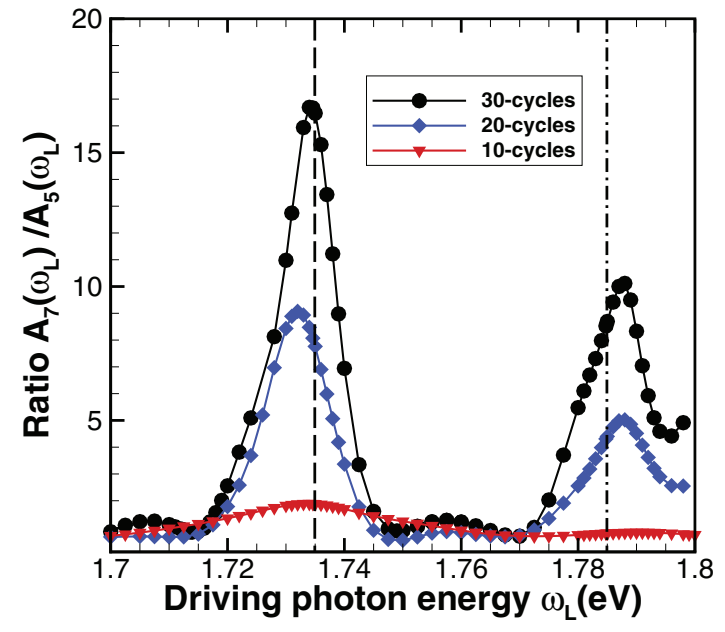

FIG. 9. (Color online) Ratio $A_{7}\left(\omega_{L}\right) / A_{5}\left(\omega_{L}\right)$ of the seventh and fifth integrated harmonic powers vs laser frequency $\omega_{L}$ for $I=$ $10 \mathrm{TW} / \mathrm{cm}^{2}$ and three laser-pulse durations. The dashed and dashdotted vertical lines mark $\omega_{a}^{\text {res }}=1.7346 \mathrm{eV}$ and $\omega_{b}^{\text {res }}=1.7848 \mathrm{eV}$, at which the seventh harmonic is resonant respectively with the $2 p 4 s\left({ }^{1} P\right)$ and $2 p 5 s\left({ }^{1} P\right)$ doubly excited states.

In Fig. 9, effects of the pulse duration on the ratio $A_{7}\left(\omega_{L}\right) / A_{5}\left(\omega_{L}\right)$ over the frequency range of $1.7-1.8 \mathrm{eV}$ are presented. We present results for three flat-top-shaped pulse lengths: $n_{c}=10-, 20-$, and 30-optical cycles (in which the laser pulse is ramped on and off over one-, two-, and eight-optical cycles, respectively). One sees in Fig. 9 that the widths of the resonance features decrease and their magnitudes increase as the pulse duration increases. For better visualization, in Figs. 10(a) and 10(b) we present the same results as in Fig. 9 on a finer energy scale in the vicinities respectively of the $2 p 4 s\left({ }^{1} P\right)$ and $2 p 5 s\left({ }^{1} P\right)$ doubly excited states. Moreover, the results for 10 and 20 cycles are normalized to those for 30 cycles. The resonance features in the case of the 10-cycle pulse, which are relatively flat in Fig. 9, are now clearly visible in Figs. 10(a) and 10(b).

The durations and bandwidths of the driving laser pulses for pulse durations of 10,20, and 30 cycles are given in Table VI for the two resonance frequencies $\omega_{a}^{\text {res }}=1.7346 \mathrm{eV}$ and $\omega_{b}^{\text {res }}=1.7848 \mathrm{eV}$, where they are compared with the widths of the resonance features in the integrated harmonic power ratio shown in Fig. 10. One observes that as the number of cycles $n_{c}$ in the laser pulse decreases, the widths $\Gamma_{n_{c}}^{a, b}$ for the resonance features in the integrated harmonic power ratio increase and are close in value to the laser-pulse bandwidths $\Delta_{n_{c}}^{a, b}$. Moreover, the widths $\Gamma_{n_{c}}^{a, b}$ are always smaller than the widths $\Gamma^{a, b}$ of the respective autoionizing states of Be. The ratio of smallness, defined as $\Gamma^{a, b} / \Gamma_{n_{c}}^{a, b}$, approximately equals $T_{n_{c}}^{a, b} / \tau^{a, b}$, the ratio of the pulse duration and the lifetime of the respective autoionizing states. In other words, as the number of cycles in the laser pulse increases, the resonance features in the spectra of integrated harmonic powers become narrower, so that the lifetime of the resonance features increases. We note that Liu et al. [71] have investigated the laser-pulse duration dependence of doubly excited resonance features in the photoabsorption spectrum of He (cf. Fig. 7 of Ref. [71]). Our present results on the pulse duration dependence of doubly 


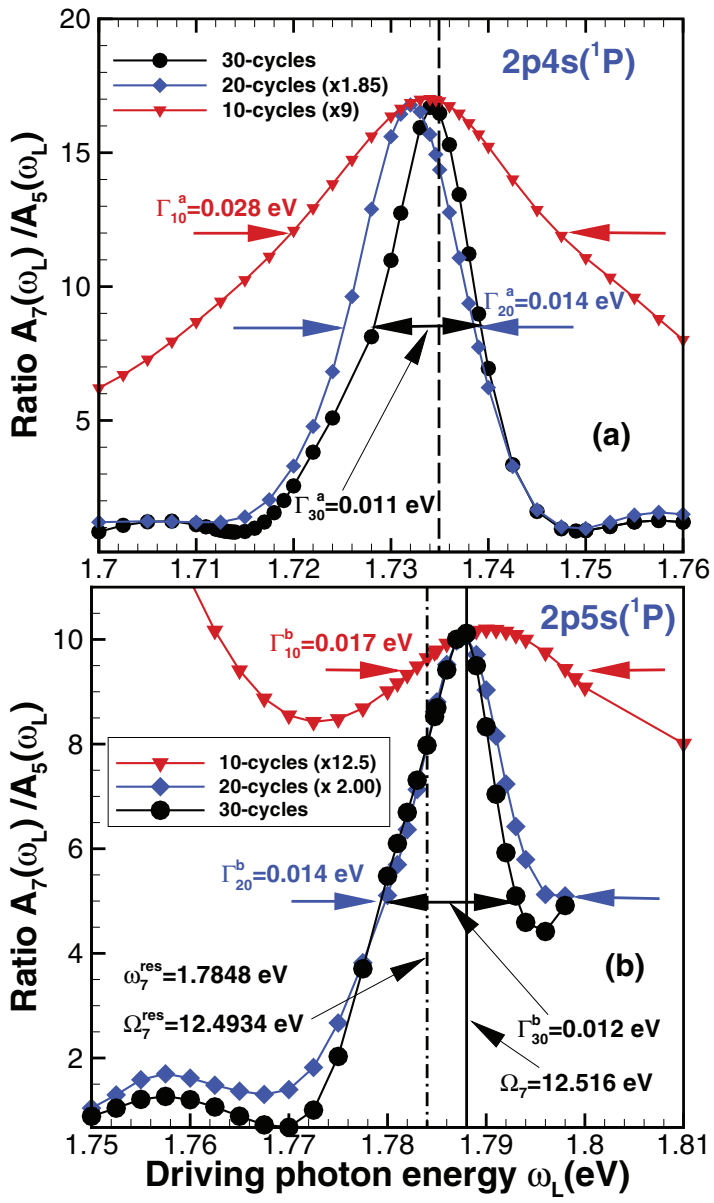

FIG. 10. (Color online) Same as in Fig. 9 but in the vicinity of (a) the $2 p 4 s\left({ }^{1} P\right)$ doubly excited state or (b) the $2 p 5 s\left({ }^{1} P\right)$ doubly excited state. The solid vertical line marks the position of the maximum achieved at $10 \mathrm{TW} / \mathrm{cm}^{2}$ in (b). Note the scale factors used in results for 10 and 20 cycles.

excited resonance features in the HHG spectrum of Be are consistent with those found in Ref. [71] for doubly excited resonances in the photoabsorption spectrum of He.

The increased magnitudes and the narrowed widths of the doubly excited state resonance features in the ratio $A_{7}\left(\omega_{L}\right) / A_{5}\left(\omega_{L}\right)$ as the driving laser-pulse duration increases (cf. Fig. 9) indicate a kind of stabilization of the doubly excited states by the driving laser pulse, similar to that termed

TABLE VI. Numerical widths, $\Gamma_{n_{c}}^{a, b}$ [cf. Figs. 10(a) and 10(b)], of resonance features in the ratio $A_{7}\left(\omega_{L}\right) / A_{5}\left(\omega_{L}\right)$ for a flat-top pulse having $n_{c}=10,20$, and 30 cycles and a peak pulse intensity of $10 \mathrm{TW} / \mathrm{cm}^{2}$. The total pulse duration $T_{n_{c}}^{a, b}$ and bandwidth $\Delta_{n_{c}}^{a, b} \simeq$ $1 / T_{n_{c}}^{a, b}$ are calculated for the resonant frequencies $\omega_{a}^{\text {res }}$ and $\omega_{b}^{\text {res }}$ at which the seventh harmonic is resonant with the (a) $2 p 4 s\left({ }^{1} P\right)$ and (b) $2 p 5 s\left({ }^{1} P\right)$ doubly excited states.

\begin{tabular}{ccccccc}
\hline \hline$n_{c}$ & $T_{n_{c}}^{a}(\mathrm{fs})$ & $\Delta_{n_{c}}^{a}(\mathrm{eV})$ & $\Gamma_{n_{c}}^{a}(\mathrm{eV})$ & $T_{n_{c}}^{b}(\mathrm{fs})$ & $\Delta_{n_{c}}^{b}(\mathrm{eV})$ & $\Gamma_{n_{c}}^{b}(\mathrm{eV})$ \\
\hline 10 & 23.8 & 0.0276 & 0.028 & 23.2 & 0.0284 & 0.017 \\
20 & 47.6 & 0.0138 & 0.014 & 46.4 & 0.0142 & 0.014 \\
30 & 71.4 & 0.0092 & 0.011 & 69.6 & 0.0095 & 0.012 \\
\hline \hline
\end{tabular}
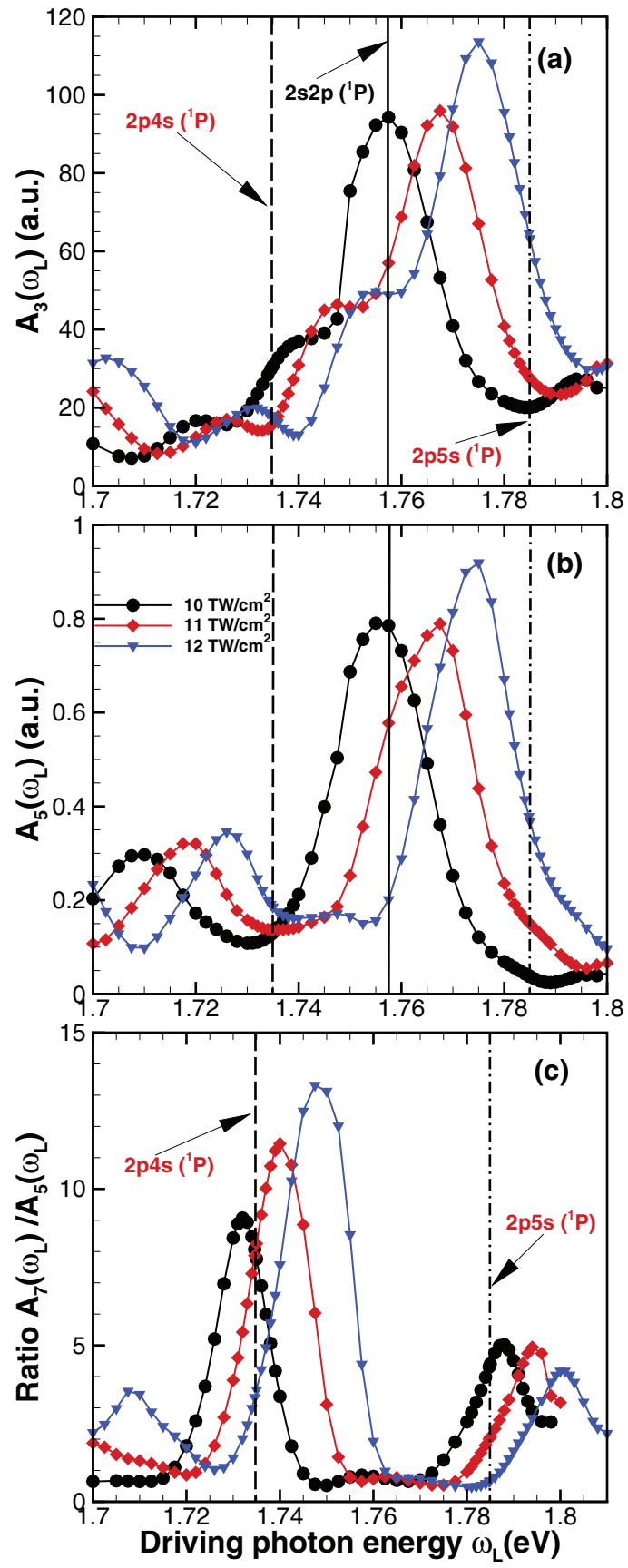

FIG. 11. (Color online) Variation of the integrated harmonic power $A_{3}\left(\omega_{L}\right)$ (a), $A_{5}\left(\omega_{L}\right)$ (b), and the ratio $A_{7}\left(\omega_{L}\right) / A_{5}\left(\omega_{L}\right)$ (c) as a function of the driving laser energy $\omega_{L}$ for three peak pulse intensities. The duration of the pulse is 20 cycles. The vertical dashed and dash-dotted lines mark $\omega_{a}^{\text {res }}=1.7346 \mathrm{eV}$ and $\omega_{b}^{\text {res }}=1.7848 \mathrm{eV}$, at which the seventh harmonic is resonant with the field-free positions of the $2 p 4 s\left({ }^{1} P\right)$ and $2 p 5 s\left({ }^{1} P\right)$ doubly excited states, respectively. The solid line marks $\omega_{L}$ at which the third harmonic is close to resonance with the field-free position of the $2 s 2 p\left({ }^{1} P\right)$ singly excited state.

the "quantum Zeno" effect [72]. Namely, as the laser-pulse duration increases, it continues to probe (or populate) the doubly excited state with which it is resonant and also stimulates harmonic emission, thus preventing autoionization of the doubly excited state. Ereifej and Story have investigated experimentally the stabilization of autoionizing states in $\mathrm{Ba}$ 

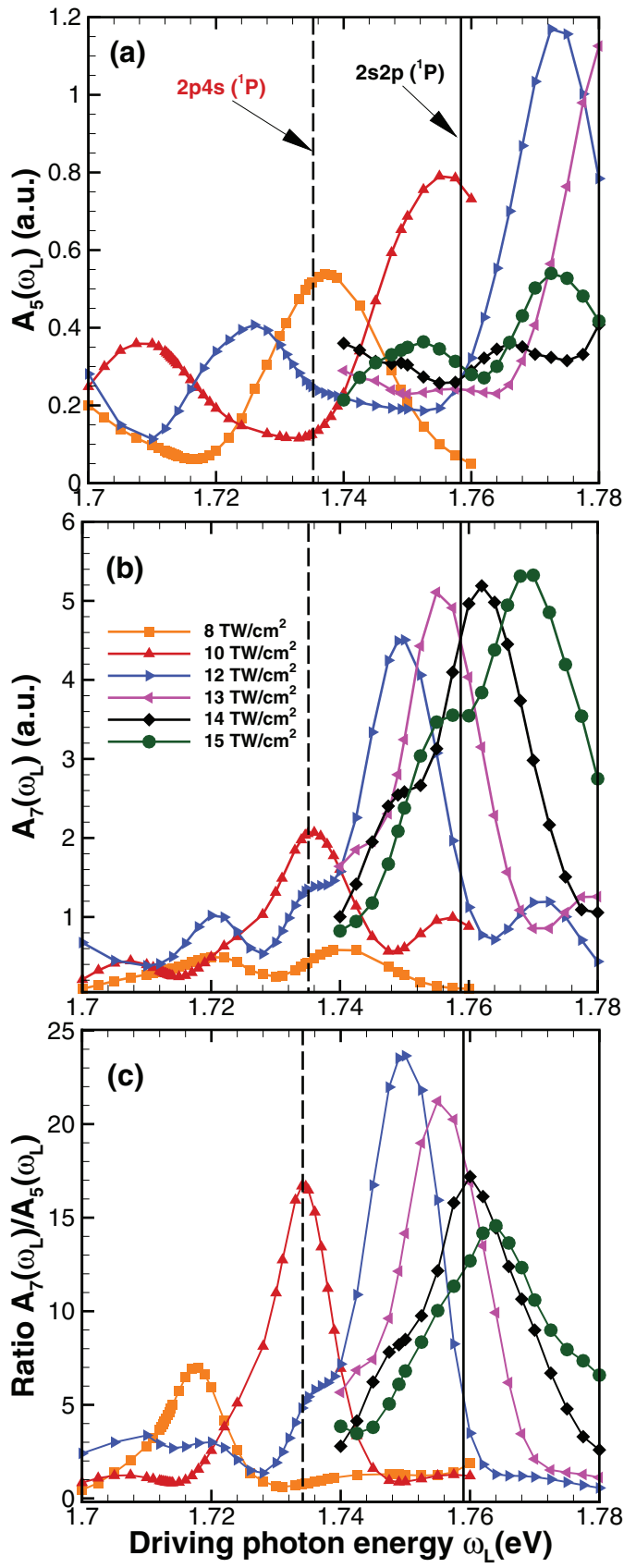

FIG. 12. (Color online) Variation of the integrated harmonic power $A_{5}\left(\omega_{L}\right)$ (a), $A_{7}\left(\omega_{L}\right)$ (b), and the ratio $A_{7}\left(\omega_{L}\right) / A_{5}\left(\omega_{L}\right)$ (c) as a function of the driving laser energy $1.7 \mathrm{eV} \leqslant \omega_{L} \leqslant 1.78 \mathrm{eV}$ for six peak pulse intensities from 8 to $15 \mathrm{TW} / \mathrm{cm}^{2}$. The duration of the pulse is 30 cycles. The dashed and solid vertical lines denote the driving laser frequencies at which there are multiphoton resonances with the field-free energies of the $2 p 4 s\left({ }^{1} P\right)$ doubly excited state and $2 s 2 p\left({ }^{1} P\right)$ singly excited state.

[73] for laser-pulse durations that were long compared to the autoionization lifetime but shorter than the fluorescence lifetimes of the autoionizing states. They showed increased stabilization against autoionization owing to laser-stimulated photoemission. In all of our results, our driving laser-pulse duration is far longer than the lifetimes of the $2 p 4 s\left({ }^{1} P\right)$ and $2 p 5 s\left({ }^{1} P\right)$ autoionizing states (cf. Tables III, V, and VI).

\section{F. Driving laser-pulse peak intensity effects on the integrated harmonic powers $A_{N}\left(\omega_{L}\right), N=3,5$, and 7}

For a 20-cycle driving laser pulse, we show in Fig. 11 how results for the integrated harmonic powers, $A_{3}\left(\omega_{L}\right)$ in Fig. 11(a), $A_{5}\left(\omega_{L}\right)$ in Fig. 11(b), and the ratio $A_{7}\left(\omega_{L}\right) / A_{5}\left(\omega_{L}\right)$ in Fig. 11(c), vary with the peak laser-pulse intensity for photon energies $1.7 \mathrm{eV} \leqslant \omega_{L} \lesssim 1.8 \mathrm{eV}$. The filled circle (black) curves are the results for $I=10 \mathrm{TW} / \mathrm{cm}^{2}$ discussed above. The other results are for two higher intensities: 11 and $12 \mathrm{TW} / \mathrm{cm}^{2}$. In Figs. 11(a) and 11(b), one sees that resonance with the $2 s 2 p\left({ }^{1} P\right)$ singly excited state is predicted for each of these intensities, with an energy shift of the $2 s 2 p\left({ }^{1} P\right)$ resonance feature that increases with increasing laser-pulse peak intensity. The ratio $A_{7}\left(\omega_{L}\right) / A_{5}\left(\omega_{L}\right)$ in Fig. 11(c) effectively removes the effect of the $2 s 2 p\left({ }^{1} P\right)$ singly excited state, and clearly shows the $2 p 4 s\left({ }^{1} P\right)$ and $2 p 5 s\left({ }^{1} P\right)$ doubly excited state resonance features, whose positions also shift to higher energies with increasing laser-pulse peak intensity.

In Fig. 12 we focus on the photon energy range in which the $2 p 4 s\left({ }^{1} P\right)$ doubly excited resonance is prominent in the integrated harmonic power $A_{7}\left(\omega_{L}\right)$. The results in this figure are for a 30-cycle trapezoidal laser pulse and for six peak pulse intensities. Results for $A_{5}\left(\omega_{L}\right)$ and $A_{7}\left(\omega_{L}\right)$ and for their ratio are shown. Whereas the $2 p 5 s\left({ }^{1} P\right)$ resonance feature has its maximum magnitude in the ratio $A_{7}\left(\omega_{L}\right) / A_{5}\left(\omega_{L}\right)$ for an intensity $\approx 10 \mathrm{TW} / \mathrm{cm}^{2}$ [cf. Fig. 11(c)], the $2 p 4 s\left({ }^{1} P\right)$ resonance feature in this ratio increases in magnitude up to an intensity of $12 \mathrm{TW} / \mathrm{cm}^{2}$ [cf. Fig. 12(c)], whereupon it decreases with further increases in peak pulse intensity. As shown by Fig. 12(b), this turning point in the intensity corresponds to a leveling off (saturation) of the magnitude of $A_{7}\left(\omega_{L}\right)$ at and beyond $12 \mathrm{TW} / \mathrm{cm}^{2}$, while the peak magnitude of $A_{5}\left(\omega_{L}\right)$ continues to increase [cf. Fig. 12(a)]. Despite the peaking of the magnitude of the $2 p 4 s\left({ }^{1} P\right)$ resonance feature in the ratio $A_{7}\left(\omega_{L}\right) / A_{5}\left(\omega_{L}\right)$, one sees in Fig. 12(c) that its energy position continues to increase with increasing laser-pulse peak intensity.

\section{SUMMARY AND CONCLUSIONS}

In summary, we have investigated the effect of resonant doubly excited states on the HHG spectrum of Be. Our focus has been on the $2 p 4 s\left({ }^{1} P\right)$ and $2 p 5 s\left({ }^{1} P\right)$ doubly excited states for driving laser frequencies in the range of seven-photon resonant transitions to these states from the ground state. Despite the fact that our laser parameters place our calculations firmly in the multiphoton regime, we predict the appearance of a plateau at the photon frequencies in seven-photon resonance with these doubly excited states. This plateau is such that the HHG power spectrum of Be increases in value by about an order of magnitude up to a cutoff harmonic of $N \approx 25$. Since this plateau is predicted in the multiphoton regime, laser-driven electron rescattering is not responsible for this plateau. Rather it originates from atomic dynamical features related to the doubly excited states as intermediate states in the harmonic-generation process.

With regard to the resonant seventh harmonic, we have shown that doubly excited state resonance features on its 
integrated harmonic power $A_{7}\left(\omega_{L}\right)$ can be isolated from lowerorder resonances with singly excited states by focusing on the ratio $A_{7}\left(\omega_{L}\right) / A_{5}\left(\omega_{L}\right)$. As the driving laser-pulse duration increases, the widths of these doubly excited state resonance features decrease and their magnitudes increase. In fact, the widths of the resonance features are comparable to those of the driving laser pulse. As the intensity of the driving laser pulse increases, the positions of the resonance features shift to higher energies. Over the range of intensities from 8 to $15 \mathrm{TW} / \mathrm{cm}^{2}$ and for a pulse duration of 30 cycles, the magnitude of the $2 p 4 s\left({ }^{1} P\right)$ resonance feature in both the integrated harmonic power $A_{7}\left(\omega_{L}\right)$ and in the ratio $A_{7}\left(\omega_{L}\right) / A_{5}\left(\omega_{L}\right)$ increase up to $\approx 12 \mathrm{TW} / \mathrm{cm}^{2}$. For higher intensities, the magnitude of $A_{7}\left(\omega_{L}\right)$ levels off, while that of the ratio decreases. The resonance feature due to resonance with the $2 p 5 s\left({ }^{1} \mathrm{P}\right)$ doubly excited state induced by a 20-cycle pulse has a maximum in the ratio at $10 \mathrm{TW} / \mathrm{cm}^{2}$ and decreases for higher intensities.

Experimental observation of our results for a Be gas seems realizable for experiments employing a spatially shaped flattop focus, as in the experiment of Toma et al. [67]. All of our predictions for the HHG spectrum, including the unexpected multiphoton regime plateau, are for driving frequencies close to the fundamental frequency of the tunable Ti:sapphire laser operating at moderate laser peak intensities. Moreover, our calculations indicate the optimal peak pulse intensities at which the doubly excited resonance features are maximal. These results for production of coherent harmonics via the double excitation of $\mathrm{Be}$ indicate the key role of electron correlations in this multiphoton regime $\mathrm{HHG}$ process.

\section{ACKNOWLEDGMENTS}

Useful discussions with John L. Hall concerning pulse duration effects are gratefully acknowledged. This work is supported in part by DOE, Office of Science, Division of Chemical Sciences, Geosciences, and Biosciences, under Grant No. DE-FG03-96ER14646. The computational results were obtained using the Merritt, Sandhills, and Tusker computational facilities at the Holland Computing Center of the University of Nebraska-Lincoln.
[1] D. Bauer, T. Brabec, H. Fehske, S. Lochbrunner, K.-H. MeiwesBroer, and R. Redmer, New J. Phys. 15, 065015 (2013), and references therein.

[2] A. McPherson, G. Gibson, H. Jara, U. Johann, T. S. Luk, I. A. McIntyre, K. Boyer, and C. K. Rhodes, J. Opt. Soc. Am. B 4, 595 (1987).

[3] M. Ferray, A. L'Huillier, X. F. Li, L. A. Lompré, G. Mainfray, and C. Manus, J. Phys. B 21, L31 (1988).

[4] P. Agostini and L. F. DiMauro, Rep. Prog. Phys. 67, 813 (2004).

[5] G. Sansone et al., Science 314, 443 (2006).

[6] V. S. Yakovlev, M. Ivanov, and F. Krausz, Opt. Express 15, 15351 (2007).

[7] E. Goulielmakis et al., Science 320, 1614 (2008).

[8] F. Krausz and M. Ivanov, Rev. Mod. Phys. 81, 163 (2009).

[9] F. Ferrari, F. Calegari, M. Lucchini, C. Vozzi, S. Stagira, G. Sansone, and M. Nisoli, Nat. Photonics 4, 875 (2010).

[10] G. Sansone, L. Poletto, and M. Nisoli, Nat. Photonics 5, 655 (2011).

[11] K. Midorikawa, Jpn. J. Appl. Phys. 50, 090001 (2011).

[12] K. Zhao, Q. Zhang, M. Chini, Y. Wu, X. Wang, and Z. Chang, Opt. Lett. 37, 3891 (2012).

[13] E. A. Gibson et al., Science 302, 95 (2003).

[14] E. J. Takahashi, T. Kanai, K. L. Ishikawa, Y. Nabekawa, and K. Midorikawa, Phys. Rev. Lett. 101, 253901 (2008).

[15] M.-C. Chen, P. Arpin, T. Popmintchev, M. Gerrity, B. Zhang, M. Seaberg, D. Popmintchev, M. M. Murnane, and H. C. Kapteyn, Phys. Rev. Lett. 105, 173901 (2010).

[16] T. Popmintchev, M.-C. Chen, P. Arpin, M. M. Murnane, and H. C. Kapteyn, Nat. Photonics 4, 822 (2010).

[17] J. Itatani, J. Levesque, D. Zeidler, H. Niikura, H. Pépin, J. C. Kieffer, P. B. Corkum, and D. M. Villeneuve, Nature (London) 432, 867 (2004).

[18] T. Morishita, A.-T. Le, Z. Chen, and C. D. Lin, Phys. Rev. Lett. 100, 013903 (2008).
[19] A.-T. Le, T. Morishita, and C. D. Lin, Phys. Rev. A 78, 023814 (2008).

[20] A.-T. Le, R. R. Lucchese, S. Tonzani, T. Morishita, and C. D. Lin, Phys. Rev. A 80, 013401 (2009).

[21] O. Smirnova, Y. Mairesse, S. Patchkovskii, N. Dudovich, D. Villeneuve, P. Corkum, and M. Yu. Ivanov, Nature (London) 460, 972 (2009).

[22] S. Haessler et al., Nat. Phys. 6, 200 (2010).

[23] H. J. Wörner, J. B. Bertrand, D. V. Kartashov, P. B. Corkum, and D. M. Villeneuve, Nature (London) 466, 604 (2010).

[24] K. J. Schafer, B. Yang, L. F. DiMauro, and K. C. Kulander, Phys. Rev. Lett. 70, 1599 (1993).

[25] P. B. Corkum, Phys. Rev. Lett. 71, 1994 (1993).

[26] M. Lewenstein, Ph. Balcou, M. Yu. Ivanov, A. L'Huillier, and P. B. Corkum, Phys. Rev. A 49, 2117 (1994).

[27] I. I. Sobel'man, Introduction to the Theory of Atomic Spectra (Pergamon Press, New York, 1972).

[28] M. V. Frolov, N. L. Manakov, T. S. Sarantseva, and A. F. Starace, J. Phys. B 42, 035601 (2009).

[29] M. V. Frolov, N. L. Manakov, T. S. Sarantseva, and A. F. Starace, Phys. Rev. A 83, 043416 (2011).

[30] M. V. Frolov, N. L. Manakov, T. S. Sarantseva, M. Yu. Emelin, M. Yu. Ryabikin, and A. F. Starace, Phys. Rev. Lett. 102, 243901 (2009).

[31] A. D. Shiner, B. E. Schmidt, C. Trallero-Herrero, H. J. Wörner, S. Patchkovskii, P. B. Corkum, J.-C. Kieffer, F. Légaré, and D. M. Villeneuve, Nat. Phys. 7, 464 (2011).

[32] R. A. Ganeev, L. B. E. Bom, J. C. Kieffer, and T. Ozaki, Phys. Rev. A 75, 063806 (2007).

[33] R. A. Ganeev, M. Suzuki, M. Baba, and H. Kuroda, Phys. Rev. A 76, 023805 (2007).

[34] M. V. Frolov, N. L. Manakov, and A. F. Starace, Phys. Rev. A 82, 023424 (2010).

[35] L. V. Keldysh, Zh. Eksp. Teor. Fiz. 47, 1945 (1964) [Sov. Phys. JETP 20, 1307 (1965)]. 
[36] X. Guan, X. M. Tong, and S.-I. Chu, Phys. Rev. A 73, 023403 (2006).

[37] I. Gilary, P. R. Kapralova-Zdanska, and N. Moiseyev, Phys. Rev. A 74, 052505 (2006).

[38] J. M. Ngoko Djiokap and A. F. Starace, Phys. Rev. A 84, 013404 (2011).

[39] A. C. Brown, D. J. Robinson, and H. W. van der Hart, Phys. Rev. A 86, 053420 (2012).

[40] A. C. Brown, S. Hutchinson, M. A. Lysaght, and H. W. van der Hart, Phys. Rev. Lett. 108, 063006 (2012).

[41] A. C. Brown and H. W. van der Hart, Phys. Rev. A 86, 063416 (2012).

[42] G. L. Kamta and A. F. Starace, Phys. Rev. Lett. 86, 5687 (2001).

[43] G. L. Kamta and A. F. Starace, Phys. Rev. A 65, 053418 (2002).

[44] S. Gilbertson, H. Mashiko, C. Li, E. Moon, and Z. Chang, Appl. Phys. Lett. 93, 111105 (2008).

[45] P. L. Altick, Phys. Rev. 169, 21 (1968).

[46] D. W. Norcross and M. J. Seaton, J. Phys. B 9, 2983 (1976).

[47] C. H. Greene, Phys. Rev. A 23, 661 (1981).

[48] R. Moccia and P. Spizzo, J. Phys. B 18, 3537 (1985).

[49] P. F. O'Mahony and C. H. Greene, Phys. Rev. A 31, 250 (1985).

[50] V. Radojević and W. R. Johnson, Phys. Rev. A 31, 2991 (1985).

[51] J. A. Tully, M. J. Seaton, and K. A. Berrington, J. Phys. B 23, 3811 (1990).

[52] H.-C. Chi, K.-N. Huang, and K. T. Cheng, Phys. Rev. A 43, 2542 (1991).

[53] B. Zhou and C. D. Lin, Phys. Rev. A 51, 1286 (1995).

[54] D.-S. Kim, S. S. Tayal, H.-L. Zhou, and S. T. Manson, Phys. Rev. A 61, 062701 (2000).

[55] G. Mehlman-Balloffet and J. M. Esteva, Astrophys. J. 157, 945 (1969).

[56] J. M. Esteva, G. Mehlman-Balloffet, and J. Romand, J. Quant. Spectrosc. Radiat. Transfer 12, 1291 (1972).
[57] G. Mehlman-Balloffet and J. M. Esteva, Space Sci. Rev. 13, 531 (1972).

[58] R. Wehlitz, D. Lukić, and J. B. Bluett, Phys. Rev. A 68, 052708 (2003).

[59] P. Olalde-Velasco, E. Méndez-Martínez, J. Jiménez-Mier, R. Wehlitz, and S. B. Whitfield, Phys. Rev. A 76, 032701 (2007).

[60] W.-C. Chu and C. D. Lin, Phys. Rev. A 82, 053415 (2010).

[61] H. Miyagi and L. B. Madsen, Phys. Rev. A 87, 062511 (2013).

[62] R. A. Ganeev, M. Suzuki, M. Baba, and H. Kuroda, J. Opt. Soc. Am. B 25, 2096 (2008).

[63] W. R. Johnson, D. Kolb, and K.-N. Huang, At. Data Nucl. Data Tables 28, 333 (1983).

[64] H. Bachau, P. Galan, and F. Martín, Phys. Rev. A 41, 3534 (1990). See the caption of Table II in this reference, in which our exponential parameter $\beta$ is defined as $\alpha$.

[65] S. Bashkin and J. O. Stoner, Atomic Energy Levels and Grotrian Diagrams (North-Holland, Amsterdam, 1975), Vol. 1. The experimental energies for $\mathrm{Be}^{+}$are also given in Table II of Ref. [64].

[66] M. Masili and A. F. Starace, Phys. Rev. A 68, 012508 (2003).

[67] E. S. Toma, Ph. Antoine, A. de Bohan, and H. G. Muller, J. Phys. B 32, 5843 (1999)

[68] J. Parker, K. T. Taylor, C. W. Clark, and S. Blodgett-Ford, J. Phys. B 29, L33 (1996).

[69] L. A. A. Nikolopoulos, T. Nakajima, and P. Lambropoulos, Eur. Phys. J. D 20, 297 (2002).

[70] M. V. Frolov, A. V. Flegel, N. L. Manakov, and A. F. Starace, J. Phys. B 38, L375 (2005).

[71] C.-N. Liu, A. Hishikawa, and T. Morishita, Phys. Rev. A 86, 053426 (2012).

[72] W. M. Itano, D. J. Heinzen, J. J. Bollinger, and D. J. Wineland, Phys. Rev. A 41, 2295 (1990).

[73] H. N. Ereifej and J. G. Story, Phys. Rev. A 60, 3947 (1999). 